\title{
Electrolyte-gated transistors for enhanced performance bioelectronics
}

\author{
Fabrizio Torricelli(i) ${ }^{\otimes}$, Demetra Z. Adrahtas (10) ${ }^{2}$, Zhenan Bao ${ }^{3}$, Magnus Berggren $^{4}$, \\ Fabio Biscarini ${ }^{5,6}$, Annalisa Bonfiglio 7 , Carlo A. Bortolotti ${ }^{5}$, C. Daniel Frisbie ${ }^{2}$, \\ Eleonora Macchia ${ }^{8}$, George G. Malliaras (10) ${ }^{9}$, Iain McCulloch (10) ${ }^{10,11}$, Maximilian Moser ${ }^{11}$, \\ Thuc-Quyen Nguyen ${ }^{12}$, Róisín M. Owens (D) ${ }^{13}$, Alberto Salleo ${ }^{14}$, Andrea Spanu (iD ${ }^{7}$ \\ and Luisa Torsi ${ }^{15}$ 凶
}

Abstract | Electrolyte-gated transistors (EGTs), capable of transducing biological and biochemical inputs into amplified electronic signals and stably operating in aqueous environments, have emerged as fundamental building blocks in bioelectronics. In this Primer, the different EGT architectures are described with the fundamental mechanisms underpinning their functional operation, providing insight into key experiments including necessary data analysis and validation. Several organic and inorganic materials used in the EGT structures and the different fabrication approaches for an optimal experimental design are presented and compared. The functional bio-layers and/or biosystems integrated into or interfaced to EGTs, including self-organization and self-assembly strategies, are reviewed. Relevant and promising applications are discussed, including two-dimensional and three-dimensional cell monitoring, ultra-sensitive biosensors, electrophysiology, synaptic and neuromorphic bio-interfaces, prosthetics and robotics. Advantages, limitations and possible optimizations are also surveyed. Finally, current issues and future directions for further developments and applications are discussed.

Bioelectronics enables the study of the aqueous media that host soft tissues and interfaces for their proper function, as well as of the connections between various cells and/or organs, which communicate by exchanging specific ions and biomolecules ${ }^{1}$. The fundamental properties of the biological systems set the requirements of the electronics counterpart. Electrolyte-gated transistors (EGTs) have emerged as important building blocks for enhanced bioelectronics because they are stable in an aqueous environment, operate at low voltages and can transduce and amplify biological signals into electronic signals ${ }^{2-5}$.

EGTs are three-terminal devices where the conductivity of a semiconducting material connected to two electrodes, classified as the source and the drain, is modulated by a third electrode known as the gate. In a basic EGT, the gate electrode and the semiconducting channel are in direct contact with the electrolyte. A voltage $V_{\mathrm{G}}$ and $V_{\mathrm{D}}$ is applied at the gate and drain electrode, respectively (FIG. 1 a). $V_{\mathrm{G}}$ and $V_{\mathrm{D}}$ are referenced to the source voltage, which is typically set to ground, $V_{\mathrm{S}}=0 \mathrm{~V}$. The polarity and magnitude of the voltage applied to the gate electrode result in a drift of cations or anions from the electrolyte to the semiconducting channel. The ionic charges can enhance or deplete the electronic charges residing in the semiconductor channel. This ionicelectronic modulation gives rise to a large variation of the channel conductivity that, in turn, manifests in a large modulation of the electronic source to drain current flowing through the transistor channel. Upon application of the gate, source and drain voltages, ions drift into the electrolyte and accumulate at the gate and semiconductor. The sign of the gate voltage controls the charge type of these ions (cations or anions), whereas its magnitude controls their density. For example, when a positive gate voltage is applied, the anion concentration increases at the gate and the cation concentration increases at the semiconducting channel. The sub-nanometre scale dimension of the ions interacting with the gate and channel materials results in a large electrostatic interaction at the gate/electrolyte and electrolyte/channel interfaces, which yields the low-voltage operation of EGTs that can range from a few volts to even less than $1 \mathrm{~V}$, depending on the specific materials used. The low-voltage operation is critically important for electrophysiology and in the case of a large variety of biosensors. In addition, in contrast to the conventional thin-film field-effect transistors, in EGTs the gate is not required to be positioned in front of the channel as the charge modulation is due to the accumulation or depletion of ions transported within 


\author{
Author addresses \\ ${ }^{1}$ Department of Information Engineering, University of Brescia, Brescia, Italy. \\ 2Department of Chemical Engineering \& Materials Science, University of Minnesota, \\ Minneapolis, MN, USA. \\ ${ }^{3}$ Department of Chemical Engineering, Stanford University, Stanford, CA, USA. \\ ${ }^{4}$ Laboratory of Organic Electronics, Department of Science and Technology, Linköping \\ University, Norrköping, Sweden. \\ ${ }^{5}$ Dipartimento di Scienze della Vita, Università degli Studi di Modena e Reggio Emilia, \\ Modena, Italy. \\ ${ }^{6}$ Center for Translational Neurophysiology of Speech and Communication, Istituto \\ Italiano di Tecnologia, Ferrara, Italy. \\ ${ }^{7}$ Department of Electrical and Electronic Engineering, University of Cagliari, Cagliari, \\ Italy. \\ ${ }^{8}$ Faculty of Science and Engineering, Åbo Akademi University, Turku, Finland. \\ ${ }^{9}$ Electrical Engineering Division, Department of Engineering, University of Cambridge, \\ Cambridge, UK. \\ ${ }^{10}$ Physical Sciences and Engineering Division, KAUST Solar Center (KSC), King Abdullah \\ University of Science and Technology (KAUST), Thuwal, Saudi Arabia. \\ ${ }^{11}$ Department of Chemistry, Chemistry Research Laboratory, University of Oxford, \\ Oxford, UK. \\ ${ }^{12}$ Department of Chemistry \& Biochemistry, University of California Santa Barbara, \\ Santa Barbara, CA, USA. \\ ${ }^{13}$ Department of Chemical Engineering and Biotechnology, University of Cambridge, \\ Cambridge, UK. \\ ${ }^{14}$ Department of Materials Science and Engineering, Stanford University, Stanford, \\ CA, USA. \\ ${ }^{15}$ Department of Chemistry, University of Bari 'Aldo Moro', Bari, Italy.
}

Bio-layer

A biological layer made with

biological entities such as, for example, antibodies, peptides, DNA, RNA, enzymes, cells, tissues or organs. the electrolyte. Therefore, in EGTs the gate can also be positioned laterally, coplanar to the source and drain electrodes, which greatly simplifies the technological fabrication process ${ }^{4,6,7}$.

EGTs are divided into two main classes depending on the ionic permeability of the channel material, which is a property related to both the electrolyte and the semiconductor. For ion-impermeable semiconductors, the ionic-electronic interaction takes place at the only electrolyte-semiconductor interface, and this mode of operation identifies electrolyte gate field-effect transistors. The ionic-electronic layer accumulated at the interface is named the electric double layer (EDL). Electrolyte gate field-effect transistors can operate with both inorganic and organic materials, including for example amorphous oxides ${ }^{8-10}$, transition metal dichalcogenides ${ }^{11,12}$, graphene ${ }^{13,14}$, nanotubes ${ }^{15-17}$, organic small molecules and polymers ${ }^{18-20}$. By contrast, the ionic-electronic interaction includes the whole three-dimensional volume of the material channel for ion-permeable semiconductors. This operational mode identifies devices addressed as electrochemical transistors $^{21-24}$. At the state of the art, ion-permeable channels are based on organic mixed ionic-electronic conductors that are often polymers, which readily solvate and transport ionic species ${ }^{25-27}$. Organic mixed ionicelectronic conductor-based EGTs give rise to the class of organic electrochemical transistors (OECTs). Internal ion-gated transistors are a class of OECTs specifically designed for bioelectronic applications based on the concept of volumetric response, where the electrolyte is embedded into an ion-conducting membrane separating the gate and the channel. Relevant state-of-art polymers used for OECTs and ion-gated transistors include poly(3,4-ethylenedioxythiophene) polystyrene sulfonate (PEDOT:PSS), poly[3(5-carboxypentyl)thiophene-2,5-diyl] (P3CPT), poly (2-(3,3'-bis(2-(2-(2-methoxyethoxy)ethoxy) ethoxy)-[2,2'-bithiophen]-5-yl)thieno[3,2-b]thiophene) (p(g2T-TT)), poly[2,6-(4,4-bis-potassium butanylsulfonate- $4 \mathrm{H}$-cyclopenta- $\left[2,1-b ; 3,4-b^{\prime}\right]$ dithiophene)-alt-4,7-(2,1,3-benzothiadiazole)] (PCPDTBT-SO ${ }_{3}$ K also known as $\mathrm{CPE}-\mathrm{K}$ ), poly(benzimidazobenzophenanthroline) (BBL) and poly[[1,2,3, 6,7,8-hexahydro-1,3,6,8-tetraoxo-2,7-bis(7-oxo3,6,9,12,15-pentaoxahexadec-1-yl)benzo[1mn][3,8] phenanthroline-4,9-diyl] [2,2'-bithiophene]-5,5'-diyl] $(\mathrm{p}(\mathrm{gNDI}-\mathrm{T} 2))^{28-32}$.

The concept of EGTs was developed by Wrighton and colleagues in 1984 using polypyrrole ${ }^{33}$, an organic ion-permeable semiconductor. Compared with ionsensitive field-effect transistors ${ }^{34-36}$ where an insulating layer separates the semiconductor and the electrolyte, the direct contact between the electrolyte and the semiconductor in EGTs enables a direct interaction between ions and electronic charges in the material channel. The class of ion-sensitive field-effect transistors ${ }^{34,36}$ are not discussed in great detail in this Primer, but are highlighted elsewhere ${ }^{37,38}$. Current efforts in EGTs leverage on the emerging materials, architectures, fabrication and integration approaches, opening possibilities for enhanced bioelectronics. Indeed, when EGTs serve in bioelectronics, the biological signals can be probed by directly embedding the medium of study within the EGT structure, such as blood, saliva, tears or lymph as the physiological liquid or cells and skin or leaf as the biological specimen.

EGT architectures. EGTs can be constructed with different device architectures, which depend on the position of the gate electrode relative to the semiconductor channel. In the top-gated geometry (FIG. 1), the gate is positioned directly over the channel. The electrolyte can be a biological fluid and its chemical, biological or ionic properties can be directly probed with the EGT. This architecture is used in electrophysiology $y^{39-41}$, ion detection ${ }^{42-44}$ and neuromorphic applications ${ }^{45-49}$. A top-gated EGT with a bio-layer embedded on the gate electrode (FIG. 1 b) is commonly used for the development of high-sensitivity label-free biosensors where protein probes such as antibodies, peptides and enzymes ${ }^{50-56}$, or genomic probes made up of DNA and RNA molecules $^{57-59}$, are linked to the gate and selectively bind and detect the target biomarker. A top-gated EGT with a biolayer placed on the top of the channel (FIG. 1c) is a typical design used for cell monitoring ${ }^{60,61}$ and in biosensors where the bio-functionalization of the semiconducting channel is deemed more convenient ${ }^{62-66}$. More insights into the self-organization and self-assembled materials used in EGTs biosensors are given in BOX 1. There is also a design where the bio-layer lacks contact with either the gate or the semiconductor surface but lies in the electrolyte (FIG. 1 d), which is applicable for monitoring the integrity of cell membrane tight junctions ${ }^{67-69}$ as well as for selective ion detection and monitoring ${ }^{70-73}$.

The less common bottom gate geometry includes an ion-conducting membrane (a solid electrolyte; shown 
Top-gated

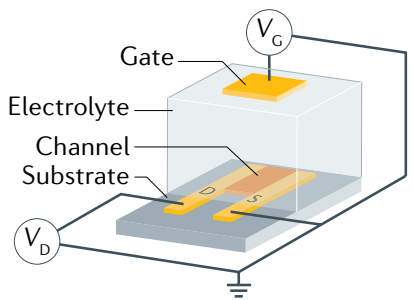

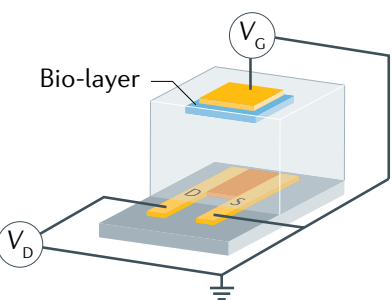

f Side-gated

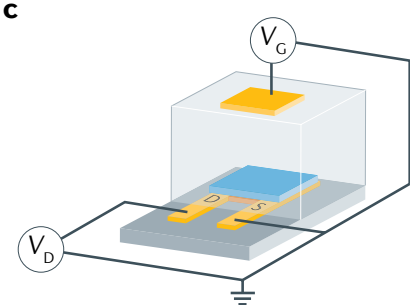

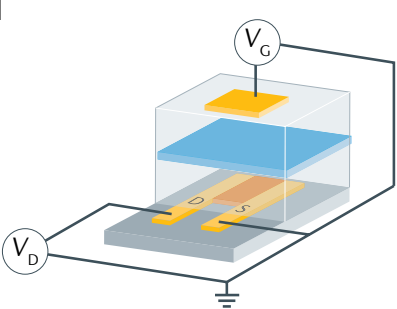

e Bottom-gated

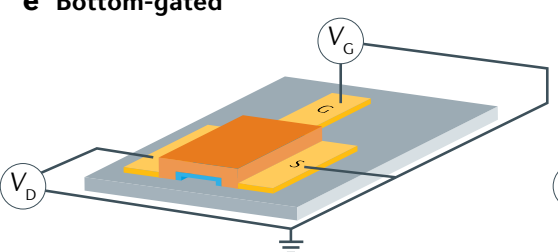

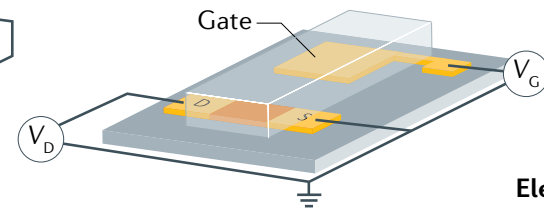

g

Extended gate (floating gate)

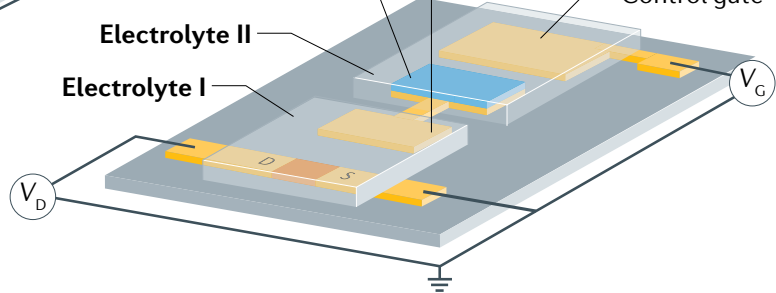

Fig. 1 | EGTs for enhanced bioelectronics. Basic architectures of an electrolyte-gated transistor (EGT). Various components, such as the gate, electrolyte, source, semiconducting channel and drain, are highlighted. $V_{\mathrm{G}}, V_{\mathrm{D}}$ and $V_{\mathrm{S}}$ are the gate, drain and source voltage, respectively. a | Top-gated architecture. $\mathbf{b}$ | Top-gated EGT with a bio-layer on the gate electrode. The gate is a polarizable electrode. c | Top-gated EGT with a bio-layer on the transistor channel. The gate can be a polarizable or non-polarizable (for example, reference) electrode. $\mathbf{d}$ | Top-gated EGT with a bio-layer included in the electrolyte. The bio-layer separates the electrolyte in two compartments. The gate can be a polarizable or non-polarizable electrode. e| Bottom-gated EGT architecture. f|Side-gated EGT architecture. $\mathbf{g}$ | Extended gate (or floating gate) EGT architecture.

in blue in FIG. 1e) that separates the metallic gate from the semiconductor channel ${ }^{74}$. This configuration can be used in physiological recording where a biocompatible semiconductor channel (PEDOT:PSS) is placed in direct contact with human tissue, for example, to record neural signals ${ }^{75}$. The gate lies in the same plane as the semiconductor channel in the side-gated geometry ${ }^{76-78}$ (FIG. 1f), which is typically used in neuromorphic applications $s^{45,79}$. In this case, the side gate can function as a global gate, simultaneously biasing several nearby semiconductor channels. A fourth EGT architecture is the extended gate (or floating gate) design (FIG. $1 \mathrm{~g}$ ), in which there are two separate electrolyte compartments (I and II) connected by a gold electrode with two pads, called the floating gate. The floating gate is capacitively coupled through the electrolytes to both the semiconductor channel and the control gate ${ }^{80}$. The floating gate geometry is commonly used for biological or chemical sensing ${ }^{81-84}$, but has also been utilized for other sensing applications, such as the transduction of cell electroactivity ${ }^{85}$. In biological sensing, capture molecules are immobilized on the arm of the floating gate in electrolyte compartment II. Target molecules introduced into compartment II bind to the functionalized pad and produce an EGT signal. The two compartments avoid direct contact of the target molecules with the semiconductor channel in compartment I, preventing possible non-specific adsorption to the semiconductor.

This Primer provides an overview of the relevant materials, technologies and experimental methods for the development of enhanced performance bioelectronics based on EGTs (Experimentation). The electrical characteristics and the meaningful device parameters of the EGTs used in bioelectronics are highlighted and examined (Results). In the Applications section, an overview of key bioelectronic applications engaging EGTs is summarized. The factors affecting the device performance and reproducibility are discussed (Reproducibility and data deposition), along with the current limitations and approaches to improve EGT performance (Limitations and optimizations). Finally, the challenges, potential new routes and future application paths are outlined (Outlook). With respect to previous reviews on EGTs covering a specific class of materials $^{4,6,86-89}$ and highlighting application-specific device implementation ${ }^{49,90-96}$, here we provide a broad perspective of EGTs for enhanced performance bioelectronics covering various aspects: from the underlying principles to best practices for experimental design, data analysis and promoting reproducibility.

\section{Experimentation}

An overview of the typical active materials used for EGTs is provided in this section, with some focus on organic semiconductors (OSCs) comprising both ion-impermeable and ion-permeable small molecules and polymers. A comprehensive overview of the organic materials used is provided in Supplementary Table 1 and the operation modes of the various classes of EGTs are given in BOX 2. Following this, inorganic 


\section{Box 1 | Self-organization and self-assembly in EGTs for bioelectronics}

The most adopted electrolyte-gated transistor (EGT) biosensor architecture envisions a large-area gate electrode functionalized with a recognition moiety ${ }^{317}$. The strategies adopted are summarized here, with special attention to those based on self-organization and self-assembly on noble metals. Gate functionalization relies on three steps: grafting a linker to the gate electrode; building a primer layer on top for anchoring the bio-recognition group; and passivating the exposed gate surface with an (antifouling) material that minimizes non-specific adsorption.

\section{Step 1: the linker}

Metal electrodes are functionalized by covalent or strong non-covalent bonds with linker-bearing molecules. The reported types of linkers and anchoring strategies are summarized in Supplementary Table 3. These approaches are simple, versatile and easy to implement, as they exploit either naturally occurring chemical functions or groups that are easy to introduce by molecular engineering.

Step 2: the primer

Three main approaches were adopted in EGTs.

a. Streptavidin or neutravidin binds biotinylated linkers on self-assembled monolayers (SAMs) on one side, and biotinylated peptides and nucleotides as bio-recognition moieties on the other side. This is a highly specific strong interaction ${ }^{318}$.

b. A monolayer of protein $\mathrm{A} / \mathrm{G}$ engineered with linkers for direct immobilization, else grafted to a SAM by formation of amide bonds. Protein $\mathrm{G}$ suitably orients antibodies with their antigen binding sites offered to the medium ${ }^{319,320}$.

c. Constructive assembly of bio-recognition molecules on SAM-forming linkers by either click chemistry or physical interactions. This approach is detailed in Supplementary Table 3.

\section{Step 3: passivation}

This is necessary to avoid uncontrolled adsorption and minimize in-parallel capacitances that may lead to signal artefacts. To fill the bare metal surface left exposed after steps 1 and 2, hydrophobic/hydrophilic SAMs ${ }^{82,321}$ or antifouling SAMs such as oligo-ethylene glycol-terminated ones ${ }^{81,322}$ are used. Alternatively, it is possible to saturate the vacancies with bovine serum albumin $(B S A)^{50,164}$.

Intermolecular hopping

events

Charge transport mechanisms taking place between various parts of molecules and polymers

Hole injection

The transfer of holes from an electrode to a semiconductor.

HOMO

(Highest occupied molecular orbital). A type of molecular orbital.

Polarons

Fermionic quasiparticles due to the strong interaction between electrons and atoms in a solid material. When electrons move in a dielectric crystal, the atoms displace from their equilibrium positions to screen the electronic charges. semiconductors made of amorphous metal oxides (AMOs) and two-dimensional materials are described and a comprehensive overview of the inorganic materials is provided in Supplementary Table 2. Finally, EGT fabrication methods based on both conventional photolithography and additive manufacturing are presented and discussed.

Organic semiconductors. OSCs used for EGTs can be clustered in two main classes: ion-impermeable OSCs that produce an EDL at the electrolyte-semiconductor interface upon application of a gate bias; and ion-permeable OSCs that generate an EDL distributed in the whole volume of the OSC ${ }^{4,6}$. The most common organic conducting material used in OECTs is PEDOT:PSS ${ }^{28,97-101}$. Originally developed as an organic conductor and used in the interlayer of photovoltaics and diodes, PEDOT:PSS has recently been used in OECTs for bioelectronics. Materials typically used for organic-based EGTs are small molecules, such as the prototypical pentacene ${ }^{102-104}$, and polymers, such as the widely used poly(3-hexylthiophene-2,5-diyl) (P3HT), poly[2,5-bis(3-tetradecylthiophen-2-yl) thieno[3,2-b]thiophene] (pBTTT-C14), poly[2,5-(2octyldodecyl)-3,6-diketopyrrolopyrrole-alt-5,5-(2,5di(thien-2-yl)thieno[3,2-b]thiophene)] (DPP-DTT) and 5,5'-bis-(7-dodecyl-9H-fluoren-2-yl)-2,2' -bithiophene (DDFTTF $)^{105-109}$. These OSCs were originally developed for organic field-effect transistors and then tuned by optimizing the processing conditions for EGT bioelectronics.
The molecular structure of the conjugated polymers and OSCs typically used for EGTs is shown in FIG. 2.

Ion-impermeable OSCs interact with the electrolyte at a single interface, resulting in a limited impact of ions on the bulk molecular structure. By contrast, in ion-permeable OSCs the hydrated ions enter inside the OSC microstructure and this additional mass can often disrupt the pristine organization of the semiconductor. This volumetric mixed ionic-electronic conduction is currently calling for new materials for the active layer in OECTs ${ }^{20,25,110}$. Several ion-permeable and ion-impermeable semiconductors are listed in Supplementary Table 1 . In both OSC types, the charge carriers are required to propagate along conjugated chains with a series of intermolecular hopping events. However, OECT semiconducting materials face other constraints imposed by aqueous and ionic ingress within the microstructure ${ }^{111-113}$. Charge injection into an OECT semiconductor, for example, can only occur when competing oxidation and reduction reactions of the aqueous media are not thermodynamically favourable. Consequently, hole injection is limited to relatively electron-rich semiconducting polymers, such as polythiophenes, and some diketopyrrolopyrrole variants, but excludes deeper HOMO (highest occupied molecular orbital) polymers. In the presence of an aqueous electrolyte, the semiconductor microstructure has to accommodate the diffusion of hydrated ions, which compensates the propagating polarons within the bulk of the semiconductor film ${ }^{112}$. Hydrated ions can disrupt the pristine organization of the semiconductor as well as introduce charge traps and coulombic pinning of the free electronic carriers. To date, semiconductors that can tolerate this physical swelling process with minimal impact on local organization seem to exhibit better performances. For example, polythiophenes with shorter glycolated side chain lengths typically outperform those with longer chain lengths, because the long chains exhibit more swelling by accommodating a larger fraction of water and ions. However, some degree of polar side chain incorporation into the polymer design is required to facilitate the hydrated ion influx. The relevant metrics for OECT electrical performance incorporate not only a carrier mobility term but also volumetric capacitance, which scales with the ionic mass density ${ }^{28}$. Long, inert, hydrocarbon chains - introduced originally in semiconducting polymers for solubility and aggregation purposes - are detrimental to capacitance. For example, in the exemplary electron-transporting polymer $\mathrm{BBL}^{114}$, large volumetric capacitances can be achieved because the polymer does not have these alkyl side chains, whereas the polystyrene sulfonate scaffold in PEDOT:PSS contributes to a much lower capacitance than analogous polythiophenes.

Conjugated polyelectrolytes (CPEs), characterized by their conjugated carbon backbone and pendant ionic side chains, are emerging as mixed ionic-electronic transport materials used in EGTs ${ }^{31,97,112,115-121}$. Focusing on CPEs, CPE-K is a self-doped polymer containing cyclopentadithiophene (CPDT) and benzothiadiazole (BT) alternating donor-acceptor units. The sulfonate side chains stabilize polarons on the conjugate backbone 
Atomic layer deposition A vacuum deposition method based on sequential use of one or more volatile compounds that react and/or decompose on the substrate surface, producing a deposit of a thin-film layer

\section{Sputtering}

A method used for the deposition of metals, insulators and semiconductors. In a vacuum chamber, a solid material (named target) is bombarded by energetic particles of a plasma or gas. The microscopic particles ejected from its surface deposit on a substrate. and the water-soluble side chains are critical for bulk doping of the film and volumetric operation. OECTs fabricated with CPE-K show accumulation-mode operation, allowing low power operation when the transistor is in its OFF state, rapid switching times and good stability over the range of temperatures relevant for biological applications ${ }^{31}$. A large maximum transconductance in a relatively small device area was obtained using interdigitated contacts that enable large channel width, making $\mathrm{CPE}-\mathrm{K}$ OECTs ideal candidates for neural interfacing. These results highlight the potential of CPEs in OECTs and suggest new optimized designs, as for example by adapting the high-mobility donor-acceptor conjugated backbones discovered by the organic field-effect transistor community to OECTs.
Inorganic semiconductors. In addition to the OSCs, various inorganic semiconductors are employed as channel materials in EGTs. Many researchers have investigated the use of amorphous oxide semiconductors (AMOs), and in some cases crystalline metal oxides, such as InGaZnO ${ }^{9122-124}, \mathrm{ZnO}^{125-128}$ or $\operatorname{In}_{2} \mathrm{O}_{3}\left(\mathrm{REFS}^{129-134}\right)$, as semiconductors in EGTs (FIG. 3a-c). These materials are generally $n$-channel materials (electron conductors) and can be deposited as thin films by various methods, including vapour-phase approaches such as atomic layer deposition or sputtering, as shown in FIG. 3g. $n$-Channel AMOs can also be solution-processed using printing methods, such as inkjet or aerosol printing, or spin-coating of inks containing molecular precursors ${ }^{126,129,130,132}$; subsequent annealing of the dried deposits at temperatures of

\footnotetext{
Box 2 | Insight into EGT operation

Upon application of a potential difference, charge polarization can be formed along semiconductor-electrolyte interfaces. Such polarization includes an elevated concentration of ions in the electrolyte and the accumulation of electronic charges within the semiconductor phase (see the figure). The former is governed by the static and dynamic fundamentals of the interfacial electric double layer (EDL), which involves an inner hydration sheet, the outer Helmholtz plane ${ }^{323}$ and a diffusive layer $^{324}$. The latter process includes the transfer of charges from a source electrode into the semiconductor, charge transport, trap-filling and a corresponding alteration of the energetic ${ }^{325}$. Efforts have been devoted to further understand the nature and details of such charge polarization, as it is crucial for the operation of electrodes and devices.

Electrochemical impedance spectroscopy, cyclic/hydrodynamic voltammetry, chrono-potentiometry/amperometry and atomic force microscopy, and transistor parameterization have been used to probe the properties of this electrolytesemiconductor interface for organic, carbon, oxide and inorganic semiconductors when interfaced with ionic liquids, aqueous or non-aqueous electrolytes ${ }^{326-331}$. In addition, modelling ${ }^{327}$ has been employed to merge the theories of electrochemistry, charge transport and solid-state (device) physics in order to describe the capacitor residing across the interface. For instance, by coupling the chemical potential of the electronic charges in the semiconductor to the EDL, the key electrical characteristics of electrolyte-gated transistors (EGTs) can be reproduced ${ }^{23}$. Better descriptions of the device parameters have been achieved for graphene-based EGTs ${ }^{332}$ and for poly(3,4-ethylenedioxythiophene) polystyrene sulfonate (PEDOT:PSS)-based capacitors by incorporating quantum capacitance into the model ${ }^{333}$. Further, the capacitor, with its included polarized charges, is defined as coplanar sheets extending only over a few nanometres in the perpendicular direction (see the figure, left) and reaches typically from a few to tens of farads per square centimetre. For mixed ion-electron and nanoporous semiconductors, the ions can penetrate the channel bulk, which causes these sheets to turn into bi-continuous networks, residing along interfaces up to hundreds of square metres per gram (see the figure, right). This creation of networks generates volumetric capacitances that can reach beyond $100 \mathrm{Fg}^{-1}$ and corresponding bulk transistor channels. For proper transistor modulation, the capacitance at the gate is equally important and the design of gate electrodes operating as polarizable or non-polarizable (faradaic) electrodes is a crucial design criterion for EGTs. To a great extent, the nature of the gate-electrolyte ${ }^{334}$ and the electrolyte-channel capacitances dictates the accumulation/depletion of charges in the channel, which defines the operational characteristics of the resulting EGT and EGT-based bioelectronics.

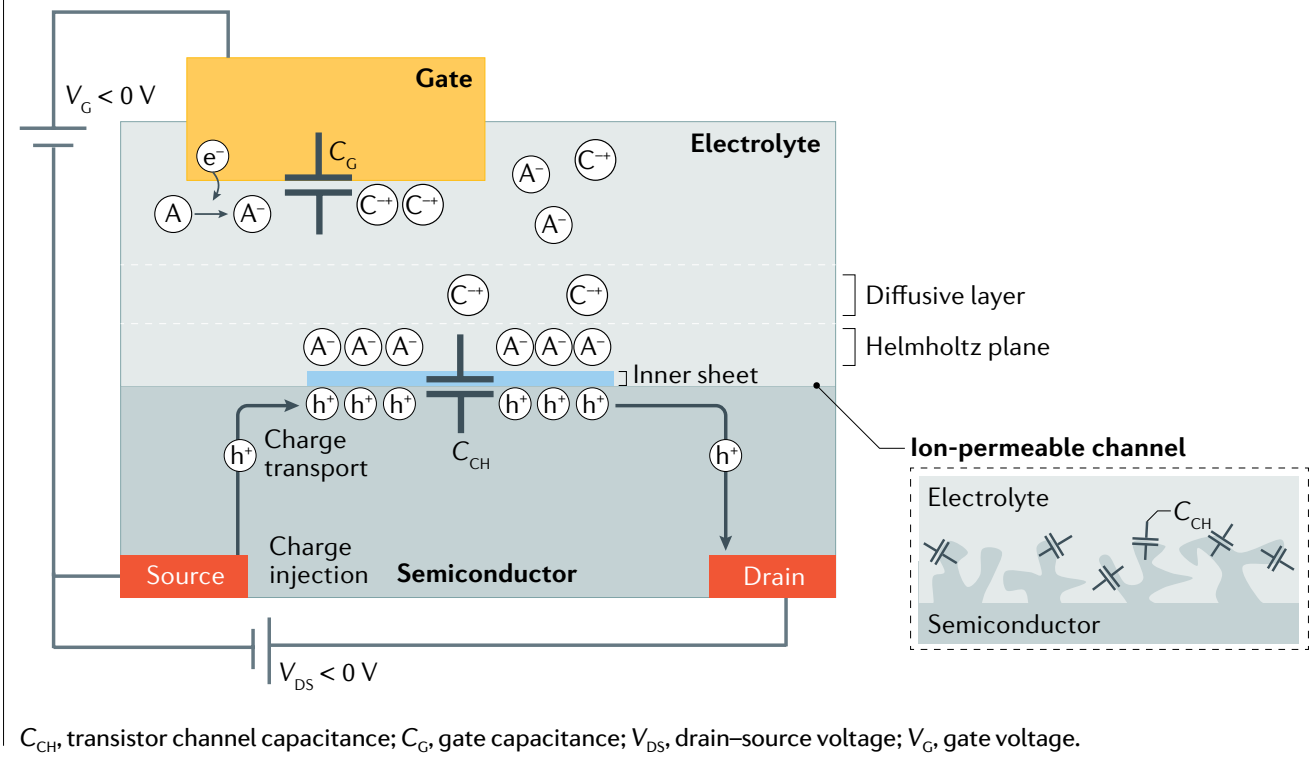




\section{Conjugated polyelectrolytes}<smiles>Cc1sc(C)c2c1COC(CO[W]#N)O2</smiles><smiles></smiles><smiles>Cc1cc(CCCCC(=O)O)cs1</smiles><smiles></smiles>

PTHS
P3CPT

\section{Conjugated polymer composites}

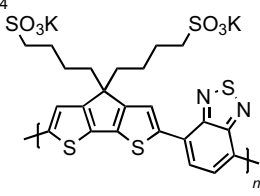

PCPDTBT-SO ${ }_{3} \mathrm{~K}$ (CPE-K)<smiles>Cc1sc(C)c2c1OCCO2</smiles>

PEDOT:PSS<smiles></smiles>

PEDOT:PSTFSILi

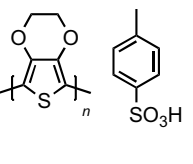

PEDOT:TOS

\section{Conjugated polymers (p-type)}

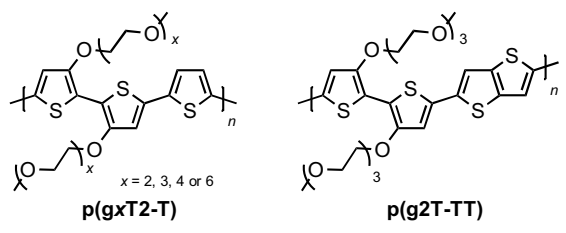

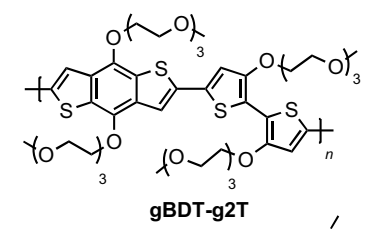

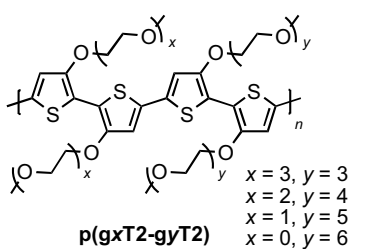<smiles>COCCN1C(=O)C2C(=O)C(C(c3ccc(-c4ccc(-c5ccc(C)s5)s4)s3)=C2c2ccc(C)s2)N1CCOC</smiles>

Conjugated polymers (n-type)

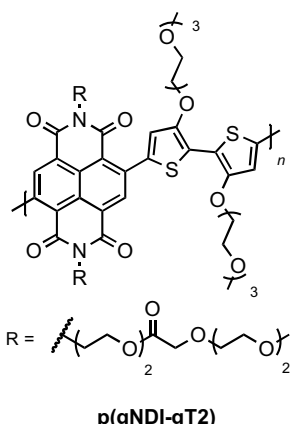

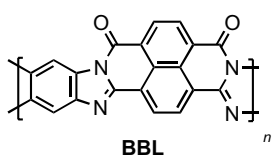

BBL

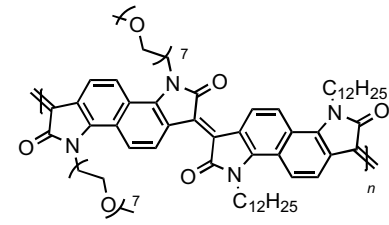

$\mathrm{PgNaN}$

Organic semiconductors<smiles>Cc1cc(C)c(P)s1</smiles>

P3HT

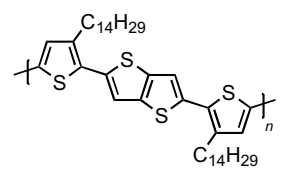

PBTTT-C14

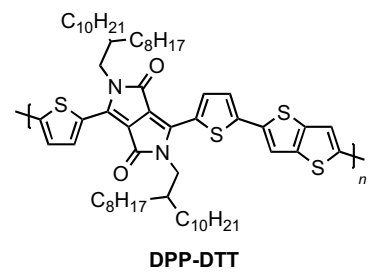

DPP-DTT

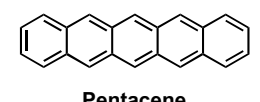

Pentacene
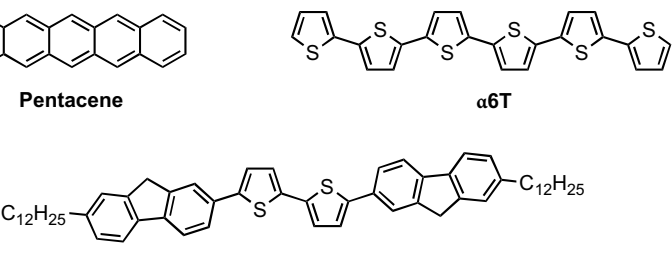

DDFTTF several hundred degrees Celsius in air produces oxide films. Advantages of AMOs include their high electron mobility, which can be well over $10 \mathrm{~cm}^{2} \mathrm{~V}^{-1} \mathrm{~s}^{-1}$, leading in principle to high gain in EGT sensing applications $s^{9,123,124}$. Moreover, AMOs can be patterned using traditional photolithographic approaches (indium-gallium-zinc oxide (IGZO) transistors are already used in industry) $)^{130}$, or with additive manufacturing strategies. An ongoing challenge with solution-processed AMOs is lowering the annealing temperatures to $200^{\circ} \mathrm{C}$ or less, which facilitates fabrication on plastic substrates ${ }^{126,129}$. The option to use plastic substrates, which is certainly possible for organic EGTs, could be important for high-throughput manufacturing of low-cost EGT biosensors.

The electrolyte gating of AMO EGTs is based on the EDL formation at the electrolyte-semiconductor interface (BOX 2). Upon application of a gate voltage, ions in the gating electrolyte generally cannot penetrate AMOs due to steric exclusion and they accumulate at the interface $^{6}$. To counter the ionic charge, oppositely charged electrons (or holes) accumulate on the AMO side of the interface, turning ON the EGT. Despite the different channel modulation mechanism between AMOs and organics, the functional result is very similar, where current increases with increasing $V_{\mathrm{G}}$ beyond a threshold gate voltage, $V_{\mathrm{T}}$.

Other alternative channel materials for EGTs include semimetals such as graphene ${ }^{58,135-138}$, two-dimensional semiconductors ${ }^{139-143}$ and carbon nanotubes ${ }^{17,144-147}$ (FIG. 3d-f). Supplementary Table 2 includes a list of the various inorganic and nanomaterial semiconductors employed in EGTs and the resulting key parameters. An advantage of two-dimensional materials or other nanomaterials is that they can be crystalline and, therefore, show large electronic mobilities ranging to as high as $50-1,000 \mathrm{~cm}^{2} \mathrm{~V}^{-1} \mathrm{~s}^{-1}$ in EGTs. Challenges compared with thin-film metal oxides include processing them by lithography or by printing, and several works are emerging on these topics ${ }^{17,141,143,148-151}$.

Fabrication approaches. Two basic approaches to the fabrication of EGTs for bioelectronics are based on photolithography ${ }^{127}$ or additive manufacturing by printing $^{80,152,153}$. EGT fabrication can be relatively simple because the gate electrode may be spatially separated from the source-drain channel. This feature eliminates 
4 Fig. 2 | Typical organic materials used for EGTs. Various classes of organic semiconductors (OSCs) developed for organic electrochemical transistors (OECTs) (conjugated polyelectrolytes (CPEs), conjugated polymer composites and conjugated polymers) and OSCs used for electrolyte-gated transistors (EGTs). $\alpha 6 \mathrm{~T}$, $\alpha$-sexithiophene; BBL, poly(benzimidazobenzophenanthroline); DDFTTF, 5,5'-bis-(7-dodecyl-9Hfluoren-2-yl)-2,2'-bithiophene; DPP-DT, poly[2,5-(2-octyldodecyl)-3,6diketopyrrolopyrrole-alt-5,5-(2,5-di(thien-2-yl)thieno[3,2-b]thiophene)]; gBDT-g2T, poly(2-(3,3'-bis(triethylene glycol monomethyl ether)-[2,2'-bithiophen]-5-yl)-(4,8bis(triethylene glycol monomethyl ether)benzo[1,2-b:4,5-b']dithiophene-2,6-diyl)); P3CPT, poly(3-carboxypentylthiophene); P3HT, poly(3-hexylthiophene); P3MEEMT, poly(3-\{[diethylene glycol monomethyl ether]methyl\}thiophene); p(gNDI-gT2), poly\{[N,N'-naphthalene-1,4,5,8-bis(dicarboximide)-2,6-diyl]-alt-5,5'-(3,3'-bis(methoxy)[2,2'-bithiophen]-5-yl)\}; pBTTT-C14, poly[2,5-bis(3-tetradecylthiophen-2-yl)thieno[3,2-b] thiophene]; PCPDTBT-SO $\mathrm{K}_{3}$, poly[2,6-(4,4-bis-potassium butanylsulfonate- $4 \mathrm{H}$ cyclopenta-[2,1-b;3,4-b']-dithiophene)-alt-4,7-(2,1,3-benzothiadiazole)]; PEDOT:PSS, poly(3,4-ethylenedioxythiophene) polystyrene sulfonate; PEDOT:PSTFSILi, poly(3,4ethylenedioxythiophene) potassium poly[4-styrenesulfonyl(trifluoromethyl sulfonyl) imide]; PEDOT-S, sodium poly(4-(2,3-dihydrothieno[3,4-b][1,4]dioxin-2-yl-methoxy)-1butylsulfonate); PEDOT:TOS, poly(3,4-ethylenedioxythiophene) tosylate; p(g3T2), poly(2-(3,3'-bis(triethylene glycol monomethyl ether)-[2,2'-bithiophen]-5-yl)); p(g0T2g6T2), poly(2-(3,3'-bis(methoxy)-[2,2'-bithiophen]-5-yl))-(2-(3,3'-bis(hexaethylene glycol monomethyl ether)-[2,2'-bithiophen]-5-yl)); p(g1T2-g5T2), poly(2-(3,3'-bis(monoethylene glycol monomethyl ether)-[2,2'-bithiophen]-5-yl))-(2-(3,3'-bis(pentaethylene glycol monomethyl ether)-[2,2'-bithiophen]-5-yl)); p(g2T2-g4T2), poly(2-(3,3'-bis(diethylene glycol monomethyl ether)-[2,2'-bithiophen]-5-yl))-(2-(3,3'-bis(tetraethylene glycol monomethyl ether)-[2,2'-bithiophen]-5-yl)); p(g2T2-T), poly(2-(3,3'-bis(diethylene glycol monomethyl ether)-[2,2'-bithiophen]-5-yl)thiophene); p(g3T2-T), poly(2-(3,3'bis(triethylene glycol monomethyl ether)-[2,2'-bithiophen]-5-yl)thiophene); $\mathrm{p}(\mathrm{g} 4 \mathrm{~T} 2-\mathrm{T})$, poly(2-(3,3'-bis(tetraethylene glycol monomethyl ether)-[2,2'-bithiophen]-5-yl) thiophene); p(g6T2-T), poly(2-(3,3'-bis(hexaethylene glycol monomethyl ether)-[2,2'bithiophen]-5-yl)thiophene); p(g2T-TT), poly(2-(3,3'-bis(triethylene glycol monomethyl ether)-[2,2'-bithiophen]-5-yl)thieno[3,2-b]thiophene); p(gDPP-T2), poly((3,6-bis(5thien-2-yl)-2,5-di(triethylene glycol monomethyl ether)-2,5-dihydropyrrolo[3,4-c] pyrrole-1,4-dione)2,2'-bithiophene); $\mathrm{PgNaN}$, poly([3,8-di-(heptaethylene glycol monomethyl ether)-3,8-dihydroindolo[7,6-g]indole-2,7-dione]-[3,8-didodecyl-1,3,6,8tetrahydroindolo[7,6-g]indole-2,7-dione]); PTEBS, sodium poly[2-(3-thienyl)-ethoxy-4butylsulfonate]; PTHS, tetrabutylammonium poly(6-(thiophene-3-yl)hexane-1-sulfonate).

Oxygen plasma

A plasma for treatment of a surface that is an effective, economical, environmentally safe method for critical cleaning.

\section{Ozone cleaning}

A cleaning process of surfaces based on photochemical

reactions involving ultraviolet light, oxygen and the material on the surface of the substrate.

Shadow masking

A technique using a metal, silicon or plastic sheet with suitably designed openings coupled to a substrate.

A material deposited over the mask can reach the substrate only in the opening regions thus defining a pattern according to the design of the mask. a difficult gate metal alignment step that is usually required for fabricating thin-film transistors in other applications ${ }^{127}$. Furthermore, the EGT channel dimensions are in the range of 5-20 $\mu \mathrm{m}$ or even larger, which also relaxes the semiconductor patterning requirements.

The fabrication approaches for building side-gated EGTs are illustrated in FIG. 4. In a typical fabrication approach (FIG. 4a), the semiconductor is first blanket deposited onto the substrate, which is followed by patterning the semiconductor into a rectangular patch using photolithography and etching. Next, a second photolithographic step includes spin-coating of photoresist, followed by light exposure through a photomask and subsequent photoresist development. In the case of the floating gate design, this second photolithographic step also produces the floating gate. Metal deposition (for example, electron beam-evaporated $50 \mathrm{~nm}$ gold, with a thin $5 \mathrm{~nm}$ titanium adhesion layer), followed by lift-off, produces the electrode pattern. These approaches can be extended also to the floating gate and charge-modulated field-effect transistor architecture (BOX 3).

In the printing approach, metals and semiconductors are deposited from liquid inks. Common printing methods for sensor fabrication include screen printing $^{76,154-156}$, inkjet printing ${ }^{153,157-159}$ and/or aerosol jet printing $^{80-83,160,161}$. FIGURE $4 \mathrm{~b}$ shows a representative process where first the metal source and drain electrodes are deposited on a substrate by screen printing. Typically, the metals need to be annealed to reach their maximum conductivity. Next, the semiconductor channel is printed ensuring alignment to the source and drain electrodes. In the case of the floating gate architecture, a third printing step is required to print a gel electrolyte over the channel and the floating gate ${ }^{80}$ (compartment I in FIG. 1g). A hybrid fabrication procedure in which conventional lithography steps are combined with printing steps can be also be adopted ${ }^{80-83,160,161}$. For example, conventional lithography can be used for the electrodes instead of screen printing in FIG. $4 \mathrm{~b}$, and printing can be used to complete the semiconductor channel and gel electrolyte. An inherent advantage of printing is the limited amount of materials needed, which can be desirable when using relatively expensive organic materials. On the other hand, lithography is extremely advanced and accessible for many laboratories. However, conventional photolithography is often incompatible with OSCs and requires either engineered resists or non-conventional processes $^{162}$.

If the EGT is being applied for selective detection of biomolecules, it is necessary to chemically functionalize the gate or the floating gate with capture molecules. Insights into bio-functionalization methods are provided in BOX 1 and Supplementary Table 3. If a gate metal electrode is functionalized, adventitious adsorbates on the gate, from processing steps such as residual photoresist, should be removed with careful cleaning using oxygen plasma or ozone cleaning. The gate electrode can be fabricated without photoresist by printing or by shadow masking of vapour-deposited metal. Functionalization of the electrode can be done by immersing the electrode in the solution ${ }^{163,164}$ or by using a PDMS well or a microfluidic cell to deliver the solution

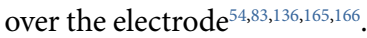

Experimental design and methods. Bio-layer integration is imperative to enable EGTs with the ability to communicate with a surrounding biological environment. A layer of biological recognition elements, such as antibodies, nucleic acids, peptides, enzymes and so on, is required for biosensors to achieve selectivity via the stable binding of markers or analytes to the elicited recognition elements ${ }^{90,163,167}$. The bio-recognition layer is anchored to one of the transistor transducing interfaces ${ }^{168}$, usually the gate or the semiconductor surface. In general, the grafting on the gate surface is more reproducible and less impactful on the device's electronic properties. For instance, functionalizing an OSC surface with anchoring chemical groups can result in lower mobilities ${ }^{169}$. FIGURE $5 a-e$ displays various strategies adopted to attach capturing molecules to a gate surface.

The physical immobilization of biomolecules involves three main approaches. For the sake of simplicity, here we focus on protein immobilization but these same considerations hold for bio-layers made of DNA, RNA and peptides. Physical absorption is achieved by direct interaction of antibodies with the solid surface (FIG. 5a), such as the gate or the semiconductor in EGTs. To improve the density, a self-assembled monolayer (SAM) can be used. Covalent immobilization can be 


\section{Polarizable}

Characterized by a charge separation at the electrodeelectrolyte interface and electrically equivalent to a capacitor

Non-polarizable

Characterized by no charge separation and electrically equivalent to a resistor obtained via a reaction between carboxyl groups immobilized on the SAM with amino groups from residues of the biomolecules (FIG. 5b). In the case of biological species bearing opposite charges, layer-by-layer absorption enables a three-dimensional structure (FIG. 5c). Bio-affinity immobilization enables the orientation of antibodies on a surface and various approaches can be adopted, which include biotinylated biomolecules captured by a streptavidin layer deposited on the electrode (FIG. 5d) or direct binding between the $\mathrm{Fc}$ region of an antibody and protein A or protein $\mathrm{G}$ deposited on the surface (FIG. 5e).

Overall, these methods can be applied to anchor the bio-layer on both the gate electrode and the semiconducting channel. Typically, gold is used for the gate electrode because it is polarizable, and there are well-established chemical methods for the grafting of a bio-layer. In the case where the bio-layer is embedded on the channel, a wide range of semiconducting and

Amorphous metal oxides

a
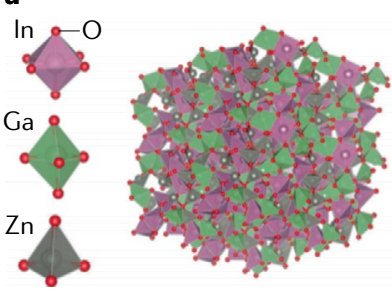

b

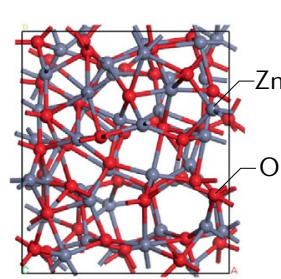

c

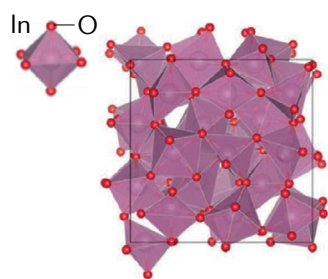

\section{Nanomaterials}

d

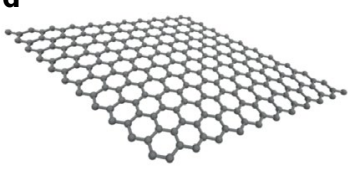

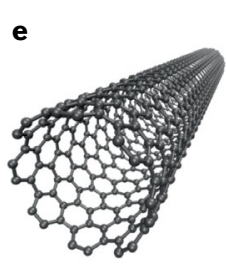

f

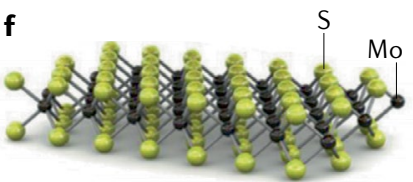

Atomic layer deposition

g

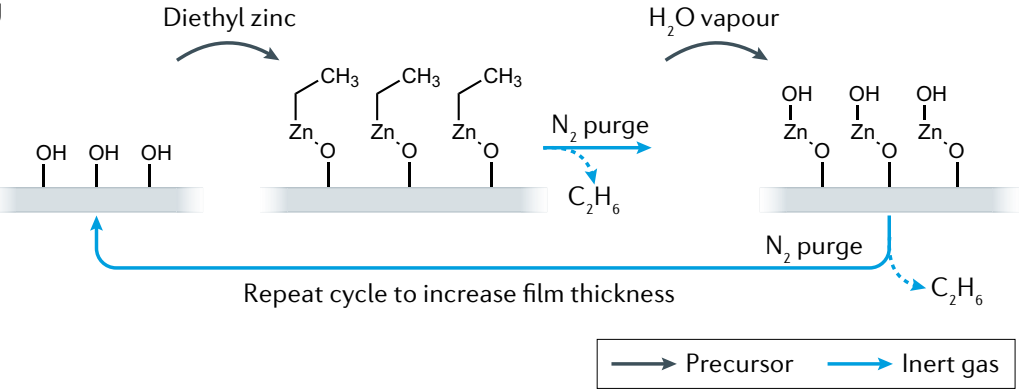

Fig. 3 | Typical inorganic semiconductors used for EGTs. a | Amorphous structure of indium-gallium-zinc oxide (IGZO). b | Structure of amorphous zinc oxide ( $\mathrm{ZnO}$ ). c|Structure of amorphous indium oxide $\left(\mathrm{InO}_{3}\right)$. $\mathbf{d} \mid$ Two-dimensional layer of the semimetal graphene. e | Single-walled carbon nanotubes. $\mathbf{f}$ | Two-dimensional molybdenum disulfide $\left(\mathrm{MoS}_{2}\right) \cdot \mathbf{g}$ | Atomic layer deposition is one method to deposit thin films of inorganic semiconductors on a substrate. Precursors injected into the chamber react with the surface of the substrate, building the film up layer by layer. Purge steps in between with inert gas remove by-products and undesired precursor from the chamber. Atomic layer deposition ultimately enables control over film thickness and uniformity. EGT, electrolyte-gated transistor. Part $\mathbf{a}$ adapted with permission from REF. ${ }^{312}$, ACS. Part $\mathbf{b}$ adapted from REF. ${ }^{313}$, CC BY 4.0 (https://creativecommons.org/licenses/by/4.0/). Part c adapted with permission from REF. ${ }^{314}$, Wiley. Parts $\mathbf{d}$ and e adapted from REF. ${ }^{315}$, CC BY 4.0 (https://creativecommons. org/licenses/by/4.0/). Part $\mathbf{f}$ adapted with permission from REF. ${ }^{316}$, Elsevier. conducting materials have been used, and the grafting method has to be properly optimized for the specific material adopted ${ }^{90,163}$. Fundamental insights into self-organization and self-assembly are given in BOX 1 and Supplementary Table 3.

\section{Results}

A fundamental advantage of EGT-based bioelectronics stems from the local bioelectric signal transduction and amplification, which results in an improved signal-to-noise ratio. The measured electrical characteristics provide meaningful information on the biological properties probed with the device. As a transistor class, the 'identity card' of EGTs is given by the transfer $\left(I_{\mathrm{D}}-V_{\mathrm{G}}\right)$ and output $\left(I_{\mathrm{D}}-V_{\mathrm{D}}\right)$ characteristics, where $I_{\mathrm{D}}$ is the drain current. An example of typical transfer and output characteristics of a hole-conducting (p-type) ion-permeable EGT and an electron-conducting (n-type) ion-impermeable EGT are displayed in FIG. $6 \mathrm{a}-\mathrm{d}$, respectively. It is important to note that the maximum applied voltages $\left(\left|V_{\mathrm{G}}\right|\right.$ and $\left.\left|V_{\mathrm{D}}\right|\right)$ should be below $1 \mathrm{~V}$ in order to prevent electrolysis of the electrolyte solution ${ }^{15,115,170}$. In addition, faradaic currents, typically displayed in a gate current $I_{G}$, should be monitored and minimized during the experiments. For instance, faradaic side reactions during the EGT operation are possible owing to redox-active materials that can produce reactive side products, which may be harmful for the local biological environment and may accelerate device degradation ${ }^{115,171}$.

EGT model and parameters. As displayed in FIG. 6e, the EGT is modelled with lumped electrical components corresponding to three main elements: a transistor channel with capacitance $C_{\mathrm{CH}}$; an electrolyte with resistance $R_{\mathrm{EL}}$; and a gate electrode with capacitance $C_{\mathrm{G}}$ connected in parallel to resistance $R_{\mathrm{G}}$. $C_{\mathrm{CH}}$ models the single or volumetric EDL capacitance, $R_{\mathrm{EL}}$ accounts for the ion movement into the electrolyte upon the application of $V_{\mathrm{G}}, C_{\mathrm{G}}$ describes polarizable gate electrodes, such as gold and platinum electrodes, and $R_{\mathrm{G}}$ describes non-polarizable gate electrodes, such as $\mathrm{Ag} / \mathrm{AgCl}$ electrodes ${ }^{91,172}$. The very same material electrode can be polarizable or nonpolarizable depending on the electrolyte used and the potential applied. Assuming there is a constant carrier mobility, $\mu$, of the electronic charges into the channel and that the EGT operates under steady-state conditions, such that transient current is vanished and $R_{\mathrm{EL}}$ can be disregarded accordingly, the drain current can be calculated. This current can be computed by solving the Poisson drift-diffusion and current continuity equations, and in the case of an electron-transporting semiconductor (n-type EGT) reads ${ }^{91,172-174}$ :

$$
\begin{aligned}
& I_{\mathrm{D}}=\frac{W}{L} \mu C\left(V_{\mathrm{G}}-V_{\mathrm{T}}-\frac{V_{\mathrm{D}}}{2}\right) V_{\mathrm{D}} \text { when } V_{\mathrm{G}}-V_{\mathrm{T}} \geq V_{\mathrm{D}} \\
& I_{\mathrm{D}}=\frac{W}{2 L} \mu C\left(V_{\mathrm{G}}-V_{\mathrm{T}}\right)^{2}\left(1+\lambda V_{\mathrm{D}}\right) \text { when } V_{\mathrm{G}}-V_{\mathrm{T}}<V_{\mathrm{D}}
\end{aligned}
$$

where $W$ and $L$ are the channel width and length, respectively, and $C=C_{\mathrm{G}} C_{\mathrm{CH}}\left(C_{\mathrm{G}}+C_{\mathrm{CH}}\right)^{-1} A_{\mathrm{CH}}{ }^{-1}$ in the case of a 


\section{a Photolithography}
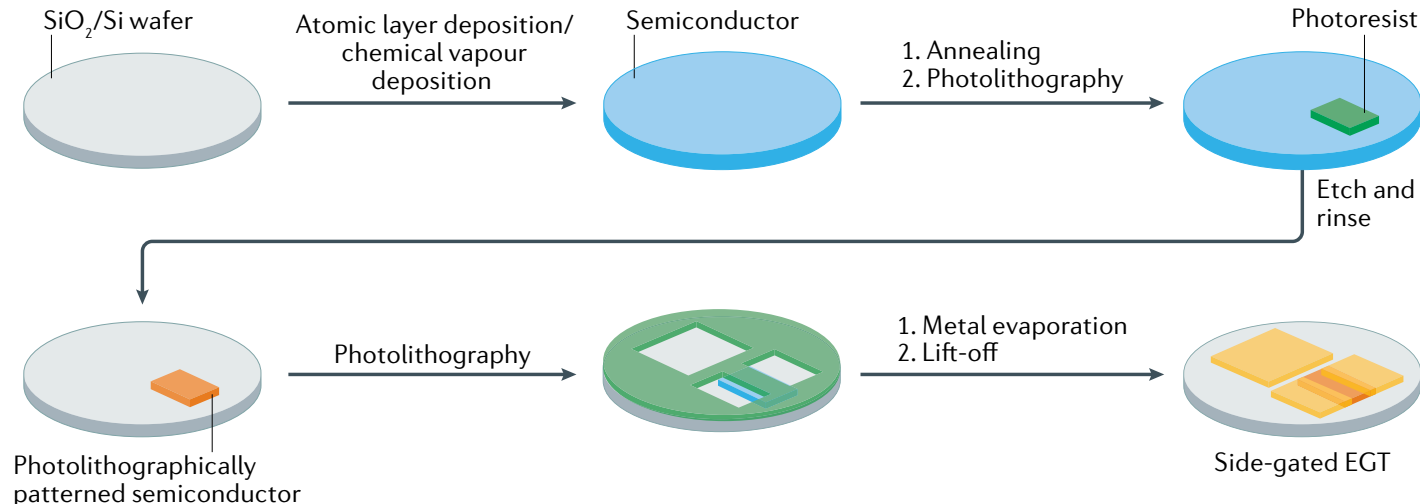

patterned semiconductor

b Printing

Cross-sectional view
Substrate (Kapton, silicon, etc.)
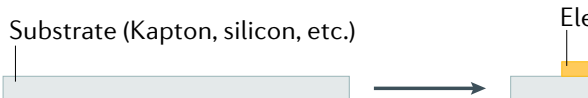

Screen printing

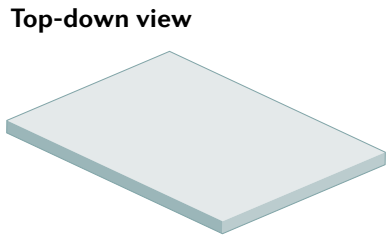

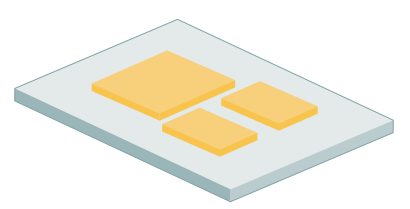
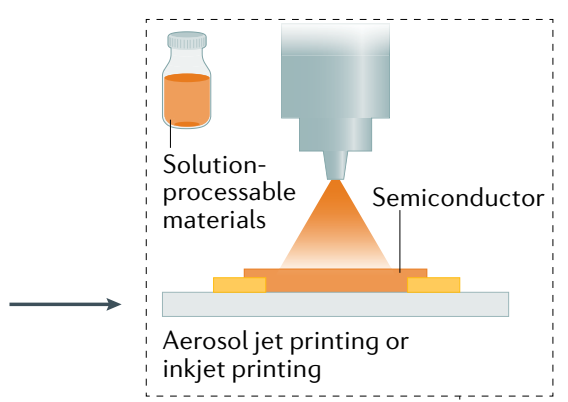

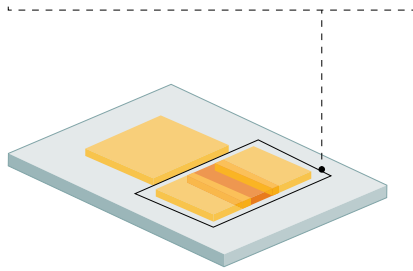

Side-gated EGT

Fig. 4 | Fabrication of EGTs. Fabrication of side-gated electrolyte-gated transistor (EGT). a | Conventional fabrication utilizing common deposition methods for semiconductor film growth and photolithography for selective patterning of the semiconductor and electrodes. Necessary equipment includes spin-coater, mask aligner, vacuum deposition chamber and vacuum evaporator chamber. Photolithography is typically performed on silicon, glass, ceramic and plastic substrates. Miniaturized devices with high-resolution features can be obtained. b | Unconventional fabrication utilizing additive processes including screen printing, aerosol jet printing and inkjet printing. Necessary equipment includes aerosol jet, inkjet, gravure and/or screen printer. Printing methods are suitable for glass, ceramic, plastic, textile and paper substrates. Fabricated devices can be flexible, wearable, on a large area and of low cost.

polarizable gate electrode and $C=C_{\mathrm{CH}} A_{\mathrm{CH}}{ }^{-1}$ in the case of a non-polarizable gate electrode. $A_{\mathrm{CH}}$ is the area of the channel; $C_{\mathrm{CH}}$ is the capacitance at the electrolyte/channel side. It is worth noting that $C_{\mathrm{CH}}=C_{\mathrm{EDL}} W L$, where $C_{\mathrm{EDL}}$ is the EDL capacitance per unit area in the case of ion-impermeable - single interface - EGTs, and $C_{\mathrm{CH}}=C_{\mathrm{V}} W L t$, where $C_{\mathrm{V}}$ is the volumetric capacitance in the case of ion-permeable EGTs and $t$ is the semiconductor thickness. Thus, the interface and volumetric capacitances can be unified considering that the overall capacitance per unit area results as $C_{\mathrm{EDL}}=C_{\mathrm{V}} t$ in the case of a volumetric capacitance EGT. $V_{\mathrm{T}}$ is the threshold accounting for various physical parameters of the materials, including, for example, the Fermi energy level of the semiconductor, the bulk ion concentration of the electrolyte, fixed charges into the polymer and the gate work function ${ }^{24,175} . \lambda$ is the channel length modulation parameter $\left(\lambda \propto L^{-1}\right)$ and accounts for the shortening of the accumulated channel when the EGT is operated at saturation. The large gating capacitance $C$, typically about $1-10010^{-6} \mathrm{~F} \mathrm{~cm}^{-2}$, results in almost flat $I_{\mathrm{D}}-V_{\mathrm{D}}$ characteristics $^{176}$ (as shown with FIG. $6 \mathrm{C}$ at $V_{\mathrm{D}}<-0.5 \mathrm{~V}$ and FIC. $6 \mathrm{~d}$ at $\left.V_{\mathrm{D}}>0.5 \mathrm{~V}\right)$. However, $\lambda$ can be neglected when the EGT is used as a bioelectronic transducer but remains a key parameter when the EGT is used in an amplifier topology $73,83,114,177-179$. Finally, Eqs. 1 and 2 can be rewritten for a p-type EGT by simply substituting $V_{\mathrm{G}}$ with $-V_{\mathrm{G}}, V_{\mathrm{D}}$ with $-V_{\mathrm{D}}$ and $I_{\mathrm{D}}$ with $-I_{\mathrm{D}}$.

Focusing on the transfer characteristics, the current $I_{\mathrm{D}}$ is modulated by $V_{\mathrm{G}}$ and the transconductance $\mathrm{g}_{\mathrm{m}}=\mathrm{d} I_{\mathrm{D}} / \mathrm{d} V_{\mathrm{G}}$ is a figure of merit quantifying the modulation efficiency and defining the intrinsic amplification ${ }^{180-182}$. $g_{\mathrm{m}}$ can be calculated directly from a transfer characteristic (FIG. 6f,g), and when the EGT operates in a linear region where $V_{\mathrm{G}}-V_{\mathrm{T}}>V_{\mathrm{D}}, g_{\mathrm{m}}$ is the slope of the $I_{\mathrm{D}}-V_{\mathrm{G}}$ curve (Eq. 1; FIG. 6a, dashed line) whereas the intersect between 
the linear least squares approximation of $I_{\mathrm{D}}$ and the $V_{\mathrm{G}}$ axis represents $V_{\mathrm{T}}$ (Eq. 1; FIC. 6a). Further, $g_{\mathrm{m}}$ depends on the bias conditions, channel geometries ( $W, L$ and $t$ ), ion species moving into the electrolyte (radius of solvated ions and ion-semiconductor interaction), bio-layer properties (geometrical dimensions, electrical, mechanical, ionic, chemical and biological properties) and EGT bioelectronic architecture. As an example, FIG. 6f,g shows $g_{\mathrm{m}}$ as a function of $V_{\mathrm{G}}$ at various $V_{\mathrm{D}}$ calculated from the $I_{\mathrm{D}}-V_{\mathrm{G}}$ displayed in FIG. $6 \mathrm{a}$ and FIG. $6 \mathrm{~b}$, respectively. The maximum $g_{\mathrm{m}}$ is of the order of hundreds or even thousands of micro-siemens $\left(g_{\mathrm{m}} / W \sim 10-100 \mathrm{Sm}^{-1}\right)$ in the case of ion-permeable EGTs ${ }^{6,180,183,184}$ (FIG. 6f) and of the order of tens of micro-siemens $\left(g_{\mathrm{m}} / W \sim 1-10 \mathrm{~S} \mathrm{~m}^{-1}\right)$ for ion-impermeable EGTs ${ }^{185-187}$ (FIG. 6g). The magnitude of $g_{\mathrm{m}}$ is a hallmark of the ionic-electronic interaction in EGTs $^{27,188-190}$ marking the large volumetric capacitance obtained in OECTs and internal ion-gated transistors.
Bioelectronic configurations. When the biological layer is integrated into the EGT architecture, various configurations can be used to transduce and enhance the bioelectronic response. FIGURE $6 h-0$ highlights various EGT-based bioelectronics used for the detection and monitoring of biological properties. More specifically, the bio-layer can be arranged on the gate electrode (FIG. 6h) or on the channel (FIG. 6j). These configurations are typically used for the detection of proteins, DNA, RNA, peptides, viruses and chemical molecules ${ }^{191-196}$, as well as for monitoring of cellular cultures ${ }^{117,147,197-199}$ and lipid bilayers ${ }^{200-202}$. The bio-layer of EGT-based biosensors is made of bio-receptors or chemical receptors specific for the target molecules being evaluated. The bio-layer embedded into the EGT is exposed to the analyte of interest, which in some cases could be directly used as the electrolyte ${ }^{5,66,203}$. To monitor cells, a layer of cells is grown or deposited on the EGT channel, and

\section{Box 3 | Insight into the charge-modulated field-effect transistor architecture for bioelectronic applications}

The organic charge-modulated field-effect transistor is a bioelectronic field-effect transistor with two gates that cooperate to set the working point, which is the bias needed to form the channel and ensure the maximization of the device's performance. The first, called the control gate, has the purpose to bias the transistor. The second, which is electrically floating, is the sensing part of the device. When one side of the extended gate (the sensing area) is functionalized and exposed to the detection environment, any charge variation within the Debye length is able to modulate the threshold voltage of the device and, therefore, the drain current. There are several strength points in this sensing strategy: the channel area is not exposed to the liquid measurement environment, preventing possible degradation of the semiconductor; the sensing surface can be specialized according to the species to detect; and the device does not need a biasing reference electrode in the solution owing to the presence of the control gate. On a planar substrate, it is possible to fabricate a large variety of materials and sensor arrays able to detect various quantities related to the same bioprocess (as depicted in the figure) including DNA-RNA detection ${ }^{335,336}$, cellular interfacing ${ }^{85,337}$, $\mathrm{pH}^{3 \text { sensing }^{338}}{ }^{33}$, temperature and pressure detection based on piezo/pyroelectric materials ${ }^{339,340}$.

This sensing strategy has the remarkable characteristic of being adaptable to any semiconductor or dielectric material. Moreover, the sensitivity of the device can be finely tuned by acting on geometric parameters, such as the ratio between the channel and the sensing area, or by adjusting the different capacitive contributions of the structure. All of these features make the organic charge-modulated field-effect transistor a versatile and ultra-sensitive tool for bioelectronic applications.

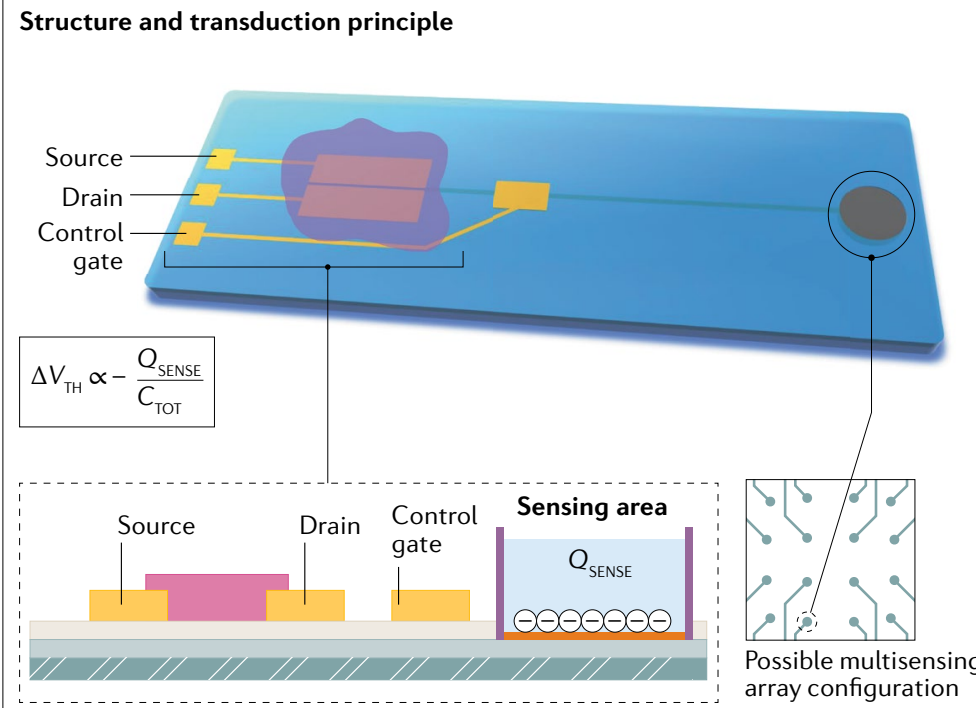

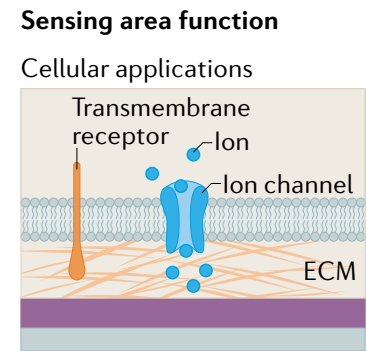

Biosensing

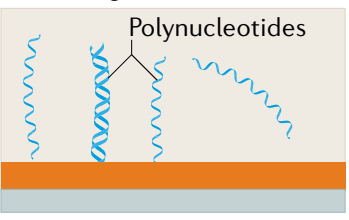

Temperature and force sensing array configuration

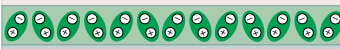

Dielectric $\square$ Floating gate metal VZ Substrate $\square$ Gold/conductive polymer

Cell adhesion layer

Biomolecule immobilization layer

- Piezo/pyroelectric material

Organic semiconductor

$C_{\mathrm{TO}}$, total capacitance; ECM, extracellular matrix; $Q_{\mathrm{SENE}}$, measured charge; $V_{\mathrm{TH}}$, threshold voltage. 


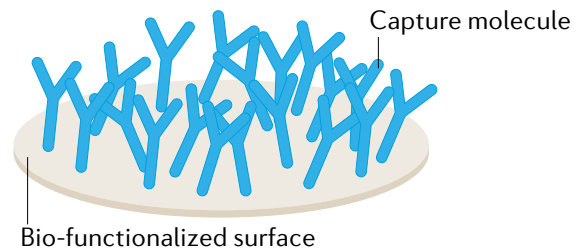

b

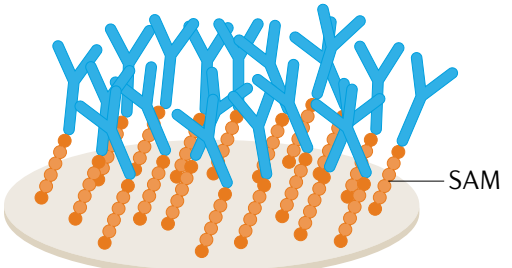

c

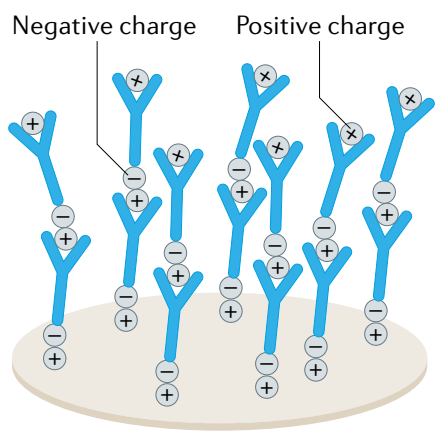

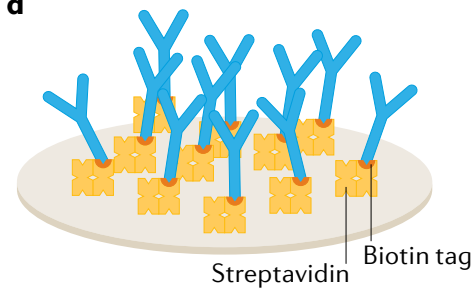

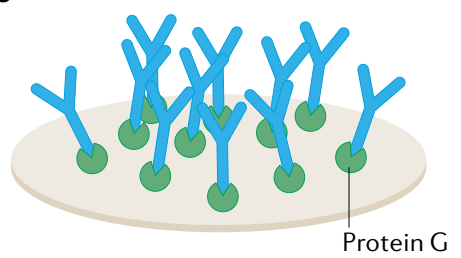

Fig. 5 | Integration of bio-layers in EGTs. a-c | Immobilization of bio-recognition elements on a solid surface by means of physical adsorption (part a), self-assembled monolayer (SAM) (part b) and stepwise deposition of biological species with opposite charges (part c). d | Bio-affinity immobilization based on biotin-tagged bio-recognition elements and streptavidin immobilized on a solid surface. e| Bio-affinity immobilization used for the orientation of biomolecules on a solid surface. EGT, electrolyte-gated transistor.

Electrocardiography The process of recording the electrical signals of the heart.

Electromyography The process of recording the electrical signals of skeletal muscles.

Electro-oculography The process of recording the electrical signals of eye. properties such as cell growth or cell differentiation processes can be tracked. When the bio-layer is positioned on the gate, a polarizable electrode is used, and $R_{\mathrm{G}}$ is large and can be neglected. By contrast, if the bio-layer is positioned on the channel, both polarizable $\left(C_{\mathrm{G}}\right.$ can be disregarded) or non-polarizable gate electrodes can be used. Typical transfer characteristics obtained when the EGT is functionalized with bio-receptors and exposed to various fluids with different analyte concentrations are displayed in FIG. 6i (REFS $\left.{ }^{50-53}\right)$. In this example, the variation of $V_{\mathrm{T}}$ as a function of the analyte concentration is displayed in a shift of the $I_{\mathrm{D}}-V_{\mathrm{G}}$ characteristics. Typical variations of $V_{\mathrm{T}}$ are of the order of tens of millivolts. These variations depend on the concentration range of the target molecule and could be attributed to the charge of the molecules detected ${ }^{50,58,204}$, Donnan's equilibrium ${ }^{205,206}$ and/or conformational changes of the bio-layer ${ }^{51,164}$. More comprehensive and systematic studies would be needed along this research direction. Depending on the position of the bio-layer, it can be electrically described as a charged capacitor in series to the gate electrode or channel. FIGURE 6k shows typical transfer characteristics obtained when cells grow on the EGT channel, where $g_{\mathrm{m}}$ lowers, resulting in a lowering of $I_{\mathrm{D}}$ in this example. The bio-layer can be modelled as a resistor $\mathrm{R}_{\mathrm{CELL}}$ in parallel to a capacitor $\mathrm{C}_{\text {CELL }}$. The $\mathrm{R}_{\text {CELL }}$ element accounts for the ion flux across the cell layers and the $\mathrm{C}_{\mathrm{CELL}}$ models the ion accumulation at the cell interfaces ${ }^{68,69,207,208}$. The lumped circuit elements describing the bio-layer $-\mathrm{R}_{\mathrm{CELL}}$ and $\mathrm{C}_{\mathrm{CELL}}-$ are connected in series to the EGT channel. Overall, EGT parameters can be affected by the biological events taking place in the bio-layer and a detailed model-based analysis can provide relevant information about the biology.

Another possible EGT bioelectronic architecture is obtained by integrating the bio-layer within the electrolyte (FIG. 6I). This topology is particularly relevant to prevent any direct interaction between the biology and the electronic material components, namely the gate and channel of the EGT. This configuration can be typically used for monitoring cell layer integrity ${ }^{67-69}$ where the cell culture is left on the Transwell filter and for selective detection of metal cations where an ion-selective membrane separates the analyte compartment from the electrolyte in contact with the EGT ${ }^{24,42-44,72,73}$. FIGURE $6 \mathrm{~m}$ shows a typical transient response obtained with an EGT without and with the cells. A voltage pulse is applied as input at the gate, and the drain current as a function of time is measured as a response. The transient response provides information on the status of the cell barriers. In more detail, without cells the EGT response is fast and rapidly achieves the steady-state output. When the barriers are embedded into the EGT, the ion flux from the gate compartment and the channel compartment depends on the status of the cell barrier. The time constant of the EGT drain current response depends on the barrier integrity and enables the quantitative monitoring of the cell barrier integrity. A detailed analysis also requires inclusion of the equivalent circuit model of the cells into the EGT lumped model shown in FIG. 6I. In a simple yet effective approach, a non-polarizable electrode is used $\left(C_{\mathrm{G}}\right.$ can be disregarded and $R_{\mathrm{G}}$ is small), and the cell layer integrated into the filter can be described with a resistor $\mathrm{R}_{\mathrm{CELL}}$ in parallel to a capacitor $\mathrm{C}_{\mathrm{CELL}}{ }^{67,147,209}$.

The EGT finds relevant application also for electrophysiology. Here, the EGT amplification enables the local amplification of the electric bio-signals, measured by directly connecting the gate and source electrodes with the tissue (as depicted in FIG. 6n). By way of example, electrocardiography, electromyography and electro-oculography with high signal-to-noise ratio can be achieved with EGTs ${ }^{75,210-213}$. In addition, the small device footprint as well as the biocompatibility of the materials enable in vivo recording of the biopotentials generated by electrogenic cells, tissues and organs $s^{2,190,214-216}$. The EGT transconductance frequency response is another relevant parameter. A typical result is displayed in FIG. 60. The signal-to-noise ratio increases by increasing 
the EGT amplification and, thus, the $g_{\mathrm{m}}$. In this respect, volumetric EGTs, namely OECTs and internal ion-gated transistors, provide superior performance owing to the enhanced volumetric response ${ }^{75,188}$. The other relevant parameter is the bandwidth of the EGT, and in the case of biopotentials the large $g_{\mathrm{m}}$ operation should extend for at least about $1,000 \mathrm{~Hz}$. We note that in conventional OECTs there is a trade-off between $g_{\mathrm{m}}$ and the bandwidth as by increasing the volumetric capacitance, $g_{\mathrm{m}}$ increases but the transistor gets slower.
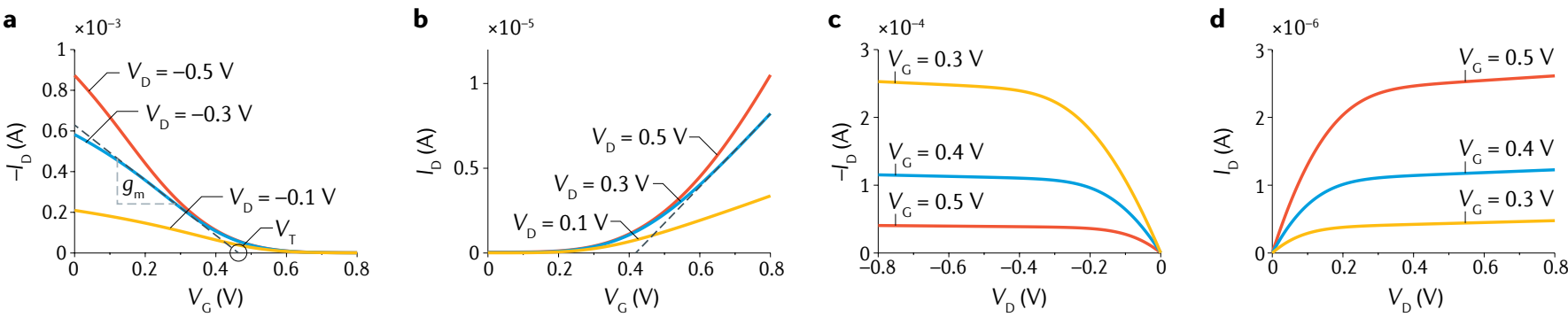

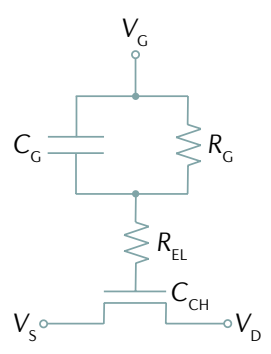

h

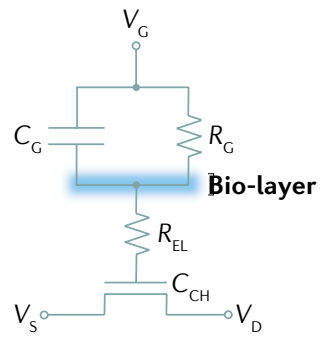

i

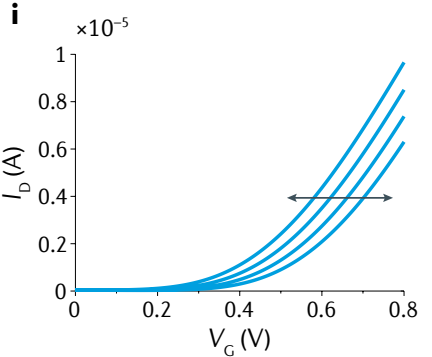

$\mathbf{g}$

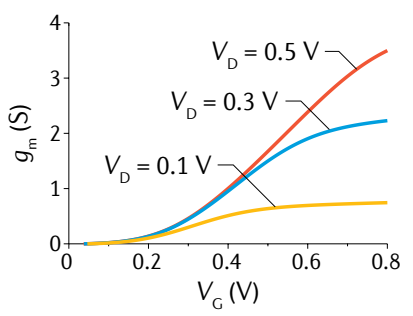

I

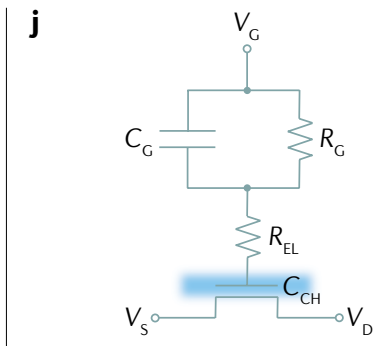

$\mathbf{k}$

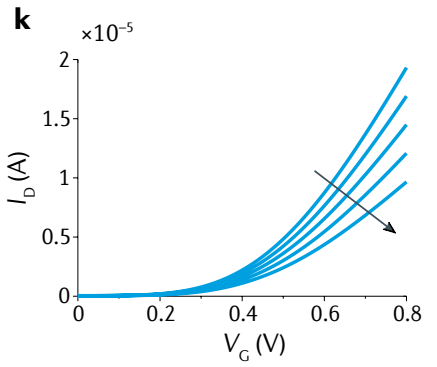

Fig. 6 | Representative electrical characteristics of EGTs. a | Typical transfer characteristics $I_{D}-V_{G}$ at various $V_{D}$ of a p-type ion-permeable and depletion-mode electrolyte-gated transistor (EGT) (full lines). Transconductance $g_{\mathrm{m}}$ and threshold voltage $V_{T}$ are highlighted (dashed line) in the case $V_{D}=-0.3 \mathrm{~V} . \mathbf{b} \mid$ Typical transfer characteristics $I_{D}-V_{G}$ at various $V_{D}$ of an n-type ion-impermeable and accumulation-mode EGT (full lines). c | Typical output characteristics $I_{D}-V_{D}$ at various $V_{G}$ of a p-type ionpermeable and depletion-mode EGT. $\mathbf{d}$ / Typical output characteristics $I_{D}-V_{D}$ at various $V_{\mathrm{G}}$ of an n-type ion-impermeable and accumulation-mode EGT. e | Schematic lumped circuit model of a bare EGT (without bio-layer). f | Typical $g_{m}$ as a function of $V_{G}$ at several $V_{D}$ in the case of a p-type ion-permeable and depletion-mode EGT. Maximum of $g_{\mathrm{m}}$ depends on both $V_{G}$ and $V_{D} \cdot \mathbf{g} \mid$ Typical $g_{m}$ as a function of $V_{G}$ at several $V_{D}$ in the case of an n-type ion-impermeable and accumulation-mode EGT. $\mathbf{h}$ | EGT lumped model highlighting the bio-layer on the gate electrode. Position of the bio-layer schematically depicted by the cyan area. $\mathbf{i} \mid$ Typical $I_{D}-V_{G}$ curves in
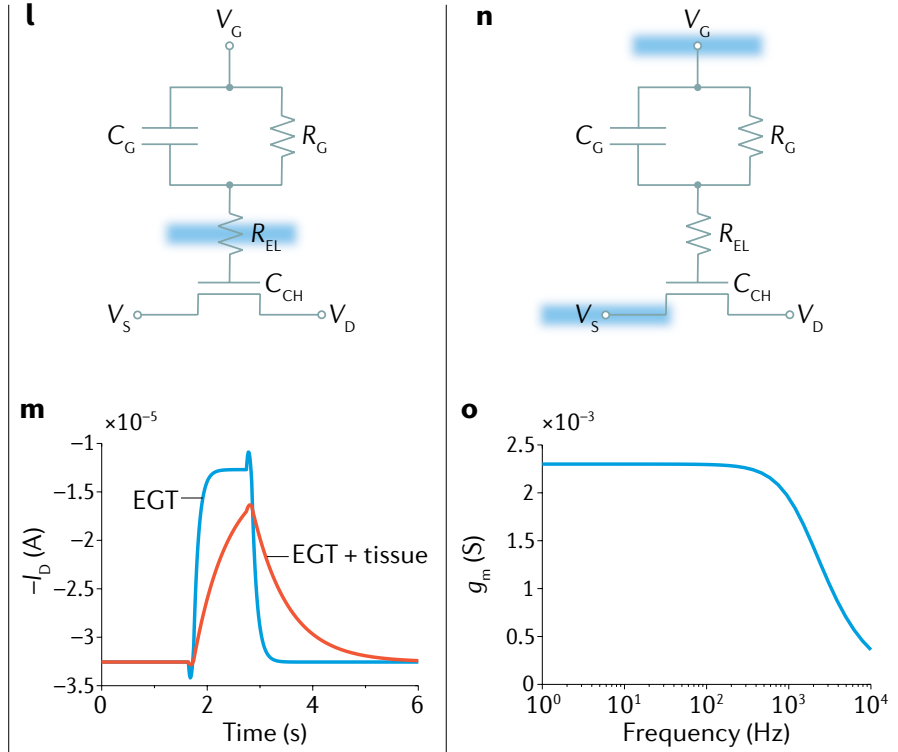

the case of selective bio-recognition taking place at the bio-layer on the gate electrode. By the way of example, these characteristics could be obtained with EGT biosensors with bio-functionalized gate. j|EGT lumped model highlighting the bio-layer on the channel. $\mathbf{k} \mid$ Typical $I_{D}-V_{G}$ curves in the case of biological events taking place on the channel mainly result in variation of bio-layer capacitance. These characteristics could be obtained when EGT is used for cell monitoring as well as EGT biosensors. I | EGT lumped model highlighting the bio-layer embedded in the electrolyte. $\mathbf{m}$ | Typical transient response obtained when the bio-layer results in a variation of the ionic resistance and/or capacitance, as for example when monitoring the cells grow and barrier integrity. $\mathbf{n}$ | EGT lumped model highlighting the external electrical connection between the EGT and the biology during electrophysiological measurements. o| Typical frequency response of an ion-permeable EGT. $C_{\mathrm{CH}}$, transistor channel capacitance; $C_{\mathrm{G}}$, gate capacitance; $I_{\mathrm{D}}$, drain current; $R_{\mathrm{EL}}$, electrolyte resistance; $R_{\mathrm{G}}$, gate resistance; $V_{D}$, drain voltage; $V_{G}$, gate voltage; $V_{S}$, source voltage. 
Spheroids

Dense three-dimensional assemblies of cells grown in gels composed of extracellular matrix.

Point-of-care Medical diagnostic testing at the place and time of the patient care.
Data analysis. To date, data analysis is based on the description of the measurements with drain current models (Eqs. 1 and 2) and simple numerical calculations displayed on the ionic-electronic EGT circuit schematic (FIG. 6e). The bio-layer is included in the EGT circuit model and routinely described as an ionic resistor in parallel to a capacitor. Then, the overall lumped circuit models can be implemented using technical programming languages such as MATLAB, Python, $\mathrm{C}++$ or other. However, to date there is a lack of standard software tools for data analysis.

\section{Applications}

In this section, we discuss relevant EGT-based bioelectronic applications including monitoring of twodimensional and three-dimensional cells, ultra-sensitive biosensors, electrophysiology, synaptic and neuromorphic interfaces, prosthetics and robotics. The applications presented highlight specific features of EGTs, including seamless three-dimensional integration with cells, wide-area biosensors with a limit of detection of $1 \pm 1$ molecules, ionic to electronic in situ transduction and amplification for local electrophysiological recording with biocompatible conformable EGTs in contact with tissues and organs, neuromorphic bio-interfaces and artificial synapses with a focus on an artificial afferent nerve.

Two-dimensional and three-dimensional cell monitoring. Cell monitoring based on electrodes has been described for several decades, where planar electrodes can be used to characterize cell adhesion, growth and differentiation ${ }^{217}$. In parallel, tissues were studied using opposing electrodes to measure resistance of a tissue and the ability to form compartments ${ }^{218}$. Multi-electrode/ transistor arrays are a third way of integrating cells with electrical monitoring ${ }^{219}$, typically recording extracellular potentials from electrogenic cells such as neurons or cardiac cells ${ }^{220}$. EGTs used for monitoring cells in vitro have followed these three trends: planar devices for monitoring cells using, for example, graphene transistors ${ }^{173}$, carbon nanotube transistors ${ }^{147}$, organic transistors with a liquid gate ${ }^{221}$ and $\mathrm{OECTs}^{222}$; opposing set-ups where the gate is separated from the source, channel and drain, for measuring trans-epithelial/endothelial resistance ${ }^{67,69}$; and multi-transistor arrays for monitoring electrogenic cells $^{223,224}$.

Early examples of integration of cells with EGTs focused on culturing cells on devices, and ensuring cytocompatibility ${ }^{225}$. There is a trade-off between the optimized device from a materials side and the biological side, and all materials exposed to the cell culture medium, which is a harsh environment for devices, must be considered. To ensure monomers or residual resists do not leach, stability experiments have to be run over time frames similar to cellular experiments, which typically take days to weeks ${ }^{226}$. In EGTs, the channel can be optically transparent allowing combined optical and electronic in situ monitoring, a property highly valued by cell biologists ${ }^{227,228}$.

Cell biology is currently focusing on growing cells in three dimensions, and also mimicking the physical environment of cells. In addition, measuring the electrical properties of cells requires intimate contact between the cell and the electrode ${ }^{229}$. Although examples have been reported of EGTs used to measure properties of three-dimensional cultures such as spheroids, the sensitivity is limited and typical EGTs will interface with only the outermost cells in the spheroid ${ }^{166}$. One interesting approach is to use bendable transistor arrays to bend around the three-dimensional shape of the tissue $\mathrm{e}^{230}$ (FIG. 7a,b). Another approach involves processing conductive polymers into three-dimensional porous scaffolds, enabling the integration of such electrodes into EGT channels ${ }^{231}$. These devices show a very slow transient response compared with microfabricated EGTs, but cells can be grown within the electrode, where individual cells can make contact with the volume of the channel $^{232,233}$ (FIG. 7b). Such configurations enable growth of complicated multicellular tissues and organs, allowing EGTs to keep pace with developments in cell biology ${ }^{234}$. The porous scaffolds can also be tuned to have mechanical properties that mimic the tissue ${ }^{235}$. Finally, the ease of mixing of biological molecules, such as extracellular matrix proteins or sugars, may enhance cell adhesion and could be used to allow spatio-temporal cell growth on a device ${ }^{236}$.

Ultra-sensitive biosensors. The ultra-sensitive detection of markers including proteins, peptides, metabolites and nucleic acids in peripheral biofluids, such as blood or saliva, can lead to advances in early diagnostic technologies ${ }^{178,237}$. Such tools are minimally invasive and suitable for point-of-care and resource-limited settings ${ }^{238}$. Single-molecule detection can be performed with either nanoscale interfaces or large millimetre wide-area interfaces.

In nanometric transducers, the device comprises a single probe that binds one of the molecules in the assayed solution. This approach can reveal a single binding event but cannot be used for the detection of a fluid encompassing markers below the picomolar concentration ${ }^{239}$. Indeed, when the marker to be detected is dispersed in a volume of $10-100 \mu \mathrm{l}$ around a nanoscale interface, an encounter between the two is very unlikely. For example, days can be needed for only a few molecules to impinge on a nanometric interface in a femtomolar concentrated solution $-\sim 10^{6}$ molecules in $100 \mu \mathrm{l}\left(\mathrm{REFS}^{237,240}\right)$.

Large-area or wide-field interfaces ${ }^{164,237}$, with their higher geometrical cross section, offer a fast (time to results of a few minutes) viable solution for molecule detection at the physical limit of a single molecule or few molecules, as shown by several examples that involve both inorganic and organic EGT devices ${ }^{241}$. FIGURE 7c shows graphene-based EGT biosensors for label-free ultra-sensitive detection of SARS-CoV-2 (REF. ${ }^{66}$ ). The EGT sensor comprises graphene sheets functionalized with the antibody that specifically binds the SARS-CoV-2 spike protein. The performance level was tested against the antigen protein, the cultured virus and nasopharyngeal swab specimens from patients with COVID-19, with limits of detection down to about 250 copies in $1 \mathrm{ml}$. Selectivity was demonstrated with MERS-CoV control experiments and patients 


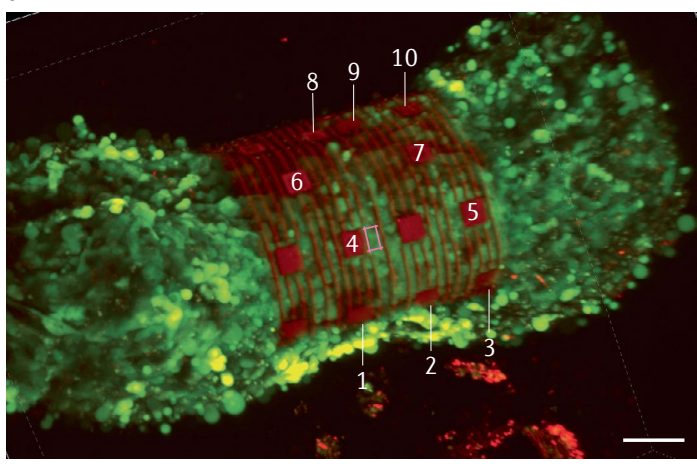

c

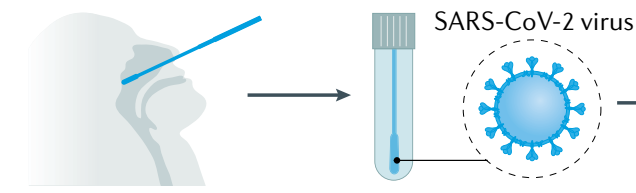

Patient with COVID-19
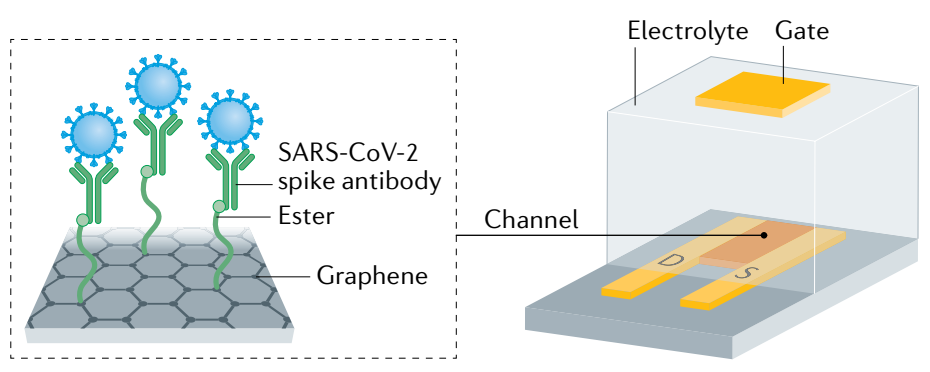

e
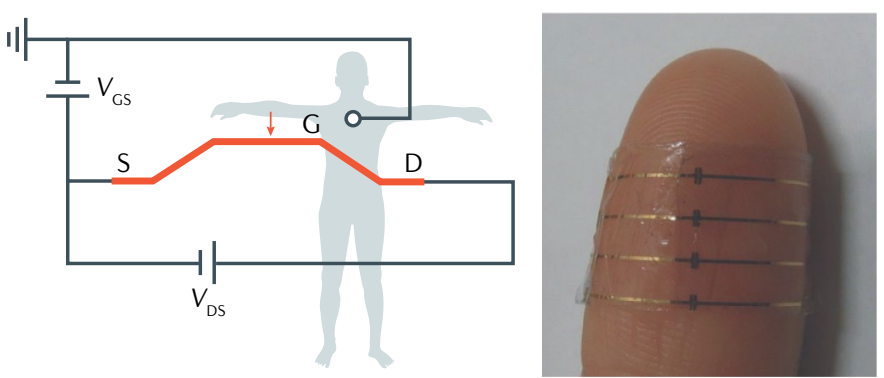

Fig. 7 | Three-dimensional cell monitoring, ultra-sensitive biosensors and in vivo electrophysiology using EGTs. a | Three-dimensional confocal microscopy image of cardiac spheroid labelled with $\mathrm{Ca}^{2+}$ indicator dye (Fluo-4, green fluorescence) encapsulated by the 3D-SR-BA. The numbers are the micro-electrodes labels. Scale bar, $50 \mu \mathrm{m}$. b|Three-dimensional conducting polymer transistors in a tube named Tubistor. The device is composed of a tubular cavity with three openings, gold-coated flexible electrodes used as source and drain contacts of the channel fixed inside the tube, and a poly(3,4-ethylenedioxythiophene) polystyrene sulfonate (PEDOT:PSS) channel. Length $L=1 \mathrm{~mm}$ and width $W=4 \mathrm{~mm}$. Gate electrode embedded inside the tube. c |COVID-19 electrolyte-gated transistor (EGT) sensor operation procedure. Graphene serves a sensing material and SARS-CoV-2 spike antibody is covalently attached to the graphene sheet via 1-pyrenebutyric acid $\mathrm{N}$-hydroxysuccinimide ester, which is an interfacing molecule serving as probe linker. $\mathbf{d}$ | Single-Molecule assay with a large-area Transistor (SiMoT) EGT transfer characteristics. Dashed grey curve corresponds to anti-human $\operatorname{lgG}$ capturing layer incubated in the bare phosphate saline buffer (PBS) solution. Same gate is further exposed, in sequence, to PBS standard solutions of $\mathrm{lg} G$ at different concentrations. f

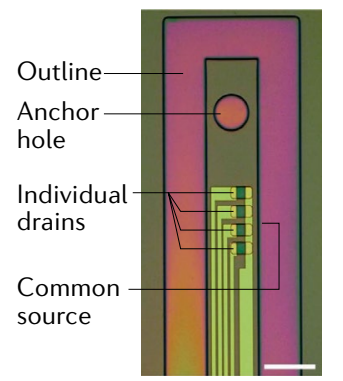

$\mathbf{g}$

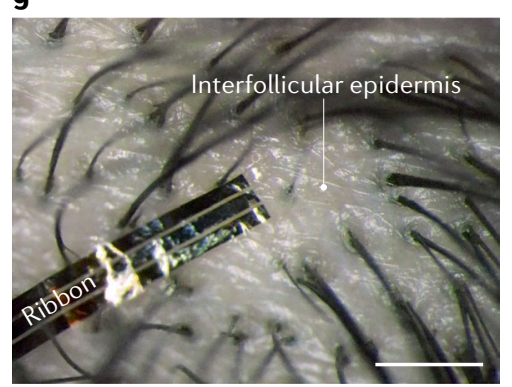

e | Wiring diagram for electrocardiographic recording with bio-resorbable EGT operated in direct contact with the skin and photograph of four organic electrochemical transistors (OECTs) showing their adaptability when attached to human skin. $\mathbf{f} \mid$ Micrograph of an implantable device with four EGTs. g| Micrograph of an EGT conforming to human scalp (scale bar, $2 \mathrm{~mm}$ ). BSA, bovine serum albumen; $I_{D}$, drain current; SAM, self-assembled monolayer; $V_{D S}$, drain-source voltage; $V_{G}$, gate voltage; $V_{G S}$, gate-source voltage; $V_{T}$, threshold voltage. Part a reprinted with permission of AAAS from REF. $^{230}$. ( T) The Authors, some rights reserved; exclusive licensee AAAS. Distributed under a CC BY-NC 4.0 License (http://creativecommons.org/ licenses/by-nc/4.0/). Part b reprinted with permission of AAAS from REF. ${ }^{233}$. (C) The Authors, some rights reserved; exclusive licensee AAAS. Distributed under a CC BY-NC 4.0 License (http://creativecommons.org/licenses/ by-nc/4.0/). Part c adapted with permission from REF. ${ }^{66}$, ACS. Part $\mathbf{d}$ adapted from REF. ${ }^{164}$, Springer Nature Limited. Part e adapted with permission from REF. ${ }^{213}$, Wiley. Part $\mathbf{f}$ adapted from REF. ${ }^{75}$, Springer Nature Limited. Part $\mathbf{g}$ reprinted with permission of AAAS from REF. ${ }^{74}$. @ The Authors, some rights reserved; exclusive licensee AAAS. Distributed under a CC BY-NC 4.0 License (http://creativecommons.org/licenses/by-nc/4.0/). 
who were both positive and negative were considered. Control experiments are essential to assess the biosensor response because other relevant effects could play a role. For example, depending on the surface properties, graphene-based EGTs can be sensitive or insensitive to the $\mathrm{pH}$ of the analyte solution ${ }^{242}$.

Single-Molecule assay with a large-area Transistor (SiMoT) technology, involving an OSC-based EGT, has enabled lowering the detection limit down to tens of zeptomoles $\left(10^{-21} \mathrm{M}\right)^{51,196,243,244}$. This method is considered a single-molecule assay as $100 \mu \mathrm{l}$ of a $10-20 \mathrm{zM}$ solution includes $1 \pm 1$ molecules ${ }^{164}$. FIGURE $7 \mathrm{~d}$ shows typical transfer characteristics measured in phosphate saline buffer (PBS) solution (red line) and in standard solutions with concentration ranging from 60 to $6 \times 10^{6} \mathrm{zM}$. In this approach, the gate electrode work function $(\phi)$ decreases at the different functionalization steps and after human IgG binding. The variation of $\phi$ is correlated to the measured threshold voltage $\left(V_{\mathrm{T}}\right)$ shift. At the physical limit, which is one molecule in the fluid assayed, the sensing was accomplished with $10^{11}-10^{12}$ recognition elements (anti-IgG) covalently attached at a millimetre-wide gate electrode and detections were possible after $10 \mathrm{~min}$ of incubation in the solution to be assayed ${ }^{239}$. SiMoT has set an unbeaten reported record in label-free single-molecule detection and the detection of proteins such as HIV-p24, CRP, IgG, IgM, peptides and genomic markers has also been demonstrated in serum ${ }^{51,164,196,243,244}$.

Electrophysiology. The electrophysiological activity of an organ and its surrounding tissue is a relevant application of EGTs in the field of health-care monitoring ${ }^{216}$. The EGT amplification has to be maximized to provide large local amplification of the bio-signals, which should be within a bandwidth of the order of $1,000 \mathrm{~Hz}$ for highfidelity and low-noise measurements. In applications where the EGT is outside the body, as for example in electrocardiography, electromyography and electrooculography, a reliable contact with the skin is essential and is typically achieved by using thin and conformable substrates. FIGURE 7e shows an array of EGTs operated in direct contact with the skin for electrocardiographic recording. In this example, the biocompatible conductive polymer PEDOT:PSS and PLGA is used for the channel and substrate, respectively. The photograph shown in FIG. 7e (REF. ${ }^{213}$ ) highlights its conformability to human skin.

Electrophysiology is also extensively used in neuroscience research and in the diagnosis of disorders of the brain, heart and muscles. Electrodes placed on the skin or implanted, such as gel-assisted $\mathrm{Ag} / \mathrm{AgCl}$ and $\mathrm{Pt} / \mathrm{Ir}$, respectively, measure signals in the brain, which can range from a few microvolts for evoked potentials to several millivolts for epileptic seizures, at frequencies of $1-1,000 \mathrm{~Hz}\left(\mathrm{REF}^{245}\right)$. Recent advances in electronics have led to a wealth of new implantable and wearable transducers for health and human performance monitoring ${ }^{246}$. EGTs are prominent here $\mathrm{e}^{39,247-249}$ because they can locally transduce and amplify the recorded signal at the source, for example when conformed to the brain surface and on organs for in vivo recording, rendering it more robust towards downstream noise. EGTs convert a voltage signal at the gate into a modulation in the drain current. The power of the signal is amplified by a factor that depends on the transconductance, which can be $>50 \mathrm{~dB}$ for a simple OECT $^{180}$.

The first application of EGTs in in vivo electrophysiology involved the use of using PEDOT:PSS OECTs to record epileptic seizures in rats. Compared with electrodes of similar size, these OECTs afforded a signalto-noise ratio more than $20 \mathrm{~dB}$ higher ${ }^{212,216}$. In a major development, the frequency response and transconductance of OECTs were significantly improved by engineering channels with internal ion reservoirs, which enabled single neuron recordings in the cortex of rats $^{75}$ (FIG. 7f). Further experiments involved arrays of PEDOT:PSS OECTs on transparent and stretchable substrates that were employed to map the heart and the brain ${ }^{250,251}$. PEDOT:PSS OECTs have also been used to stimulate cortical neurons ${ }^{252}$. A similar path was followed for graphene-based EGTs, where individual devices ${ }^{253}$, as well as passive and actively multiplexed arrays ${ }^{40,254-257}$, were used to record and map cortical activity. Other notable examples include brain recordings made with injectable nanowire transistors ${ }^{258}$ and electrolyte-gated metal oxide semiconductor field-effect transistors ${ }^{259}$.

EGTs were first applied in cutaneous electrophysiology to measure cardiac activity using a PEDOT:PSS OECT that was attached to the skin of volunteers with the aid of a gel ${ }^{213}$. The use of non-volatile gels enabled stable recordings over several days ${ }^{260}$. A novel organic EGT geometry based on conducting fibres encapsulated in a soft biodegradable polymer was integrated with textiles and used to record cardiac activity ${ }^{261}$. The high transconductance of OECTs with internal ion reservoirs allowed miniaturization and direct attachment between hair follicles for long-term measurements of brain activity, as displayed in FIG. $7 \mathrm{~g}\left(\mathrm{REF}^{74}\right)$. A different method of coupling EGTs to the body involves the use of a cutaneous electrode as the gate, which has afforded applications of PEDOT:PSS OECTs in electrocardiography, electro-oculography and electroencephalography ${ }^{262}$. Tuning the thickness of the channel was used to navigate the gain versus bandwidth trade-off ${ }^{188}$.

Compared with electrodes, which show high impedance at low frequencies, the low-pass filter characteristics of EGTs make them particularly suitable for electrophysiology recordings and transducer miniaturization. The key issues with EGTs include the high power consumption, and the fact that the signal is in the form of a current and, thereby, incompatible with standard electrophysiology amplifiers. However, encouragingly, an improved power consumption was observed when an EGT was operated in a sub-threshold regime ${ }^{263}$. Moreover, a simple circuit with a drain resistor was reported as an efficient method to convert the output current into voltage $^{177}$. Despite these reports, there is still room for creative solutions on these fronts.

Synaptic and neuromorphic bio-interfaces. Neuromorphic EGTs are an emerging platform for synaptic electronics because EGTs offer large capacitance (up to $500 \mu \mathrm{F} \mathrm{cm}^{-2}$ ) for efficiently coupling the gate to the channel ${ }^{264-266}$. The large and tunable ionic-electronic 


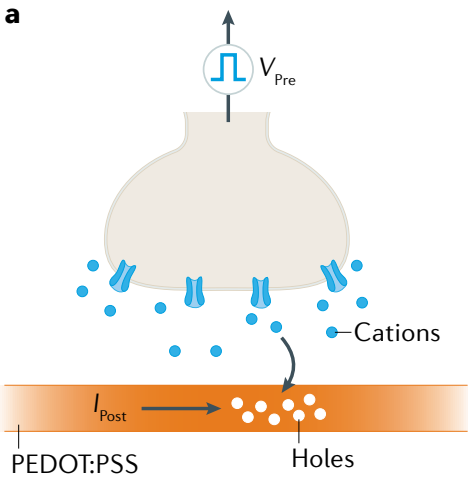

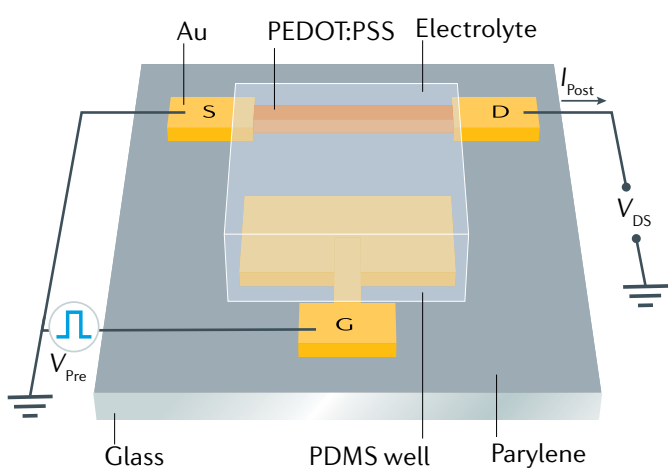

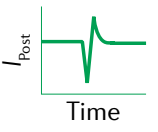

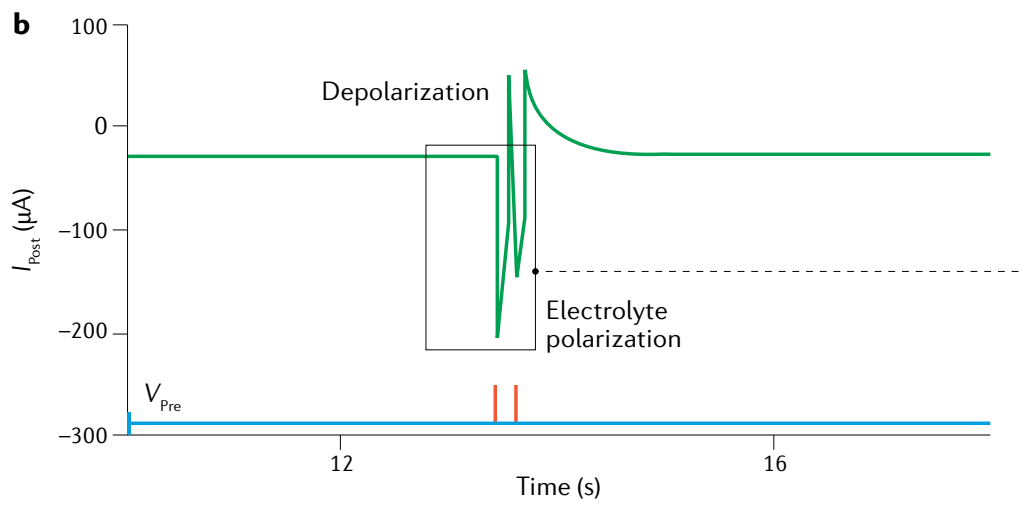
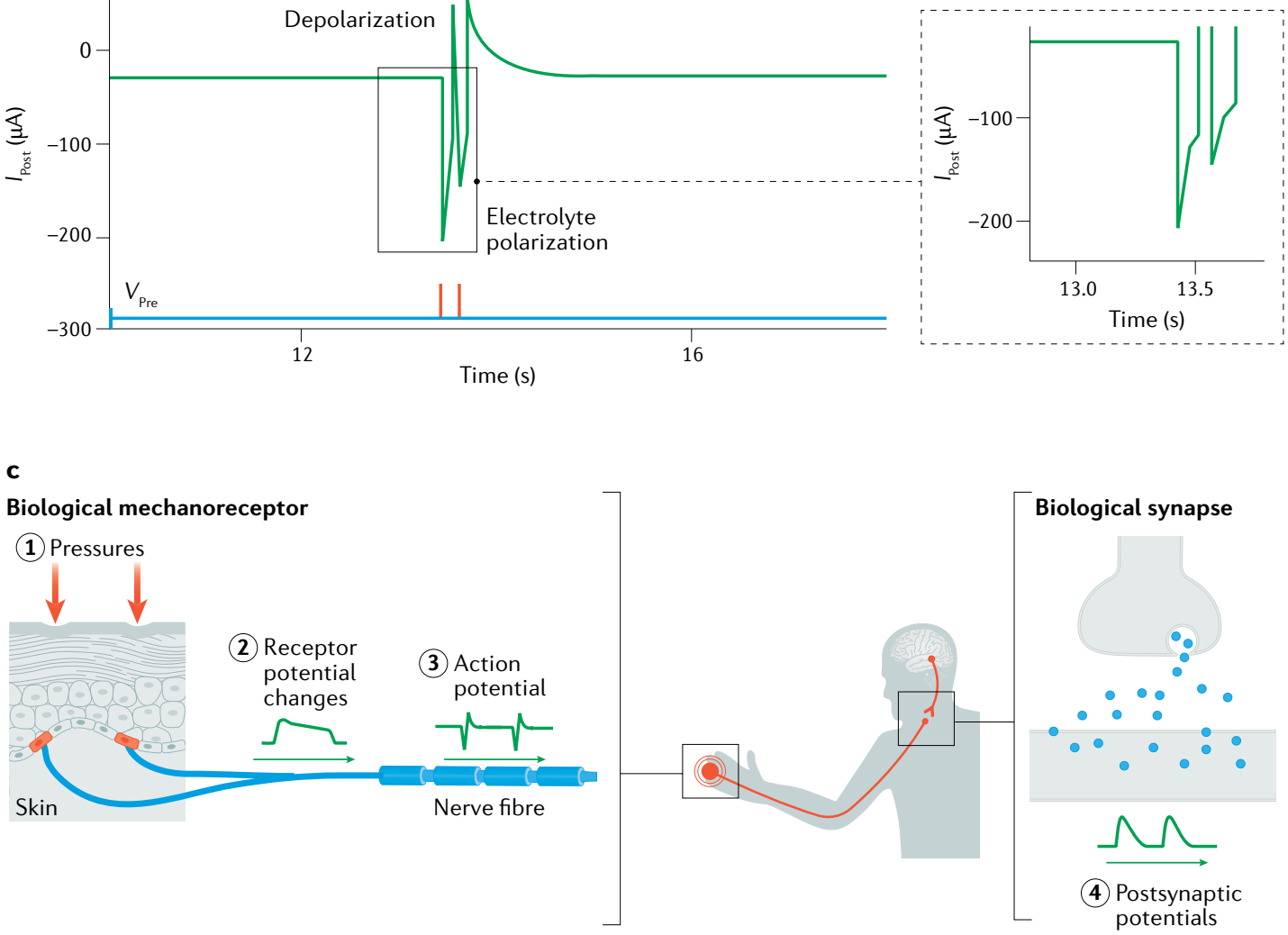

d

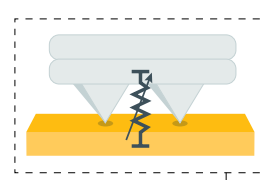

(1) Pressures

(2) Voltage

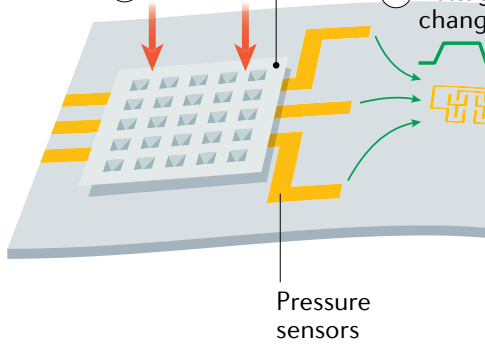

changes

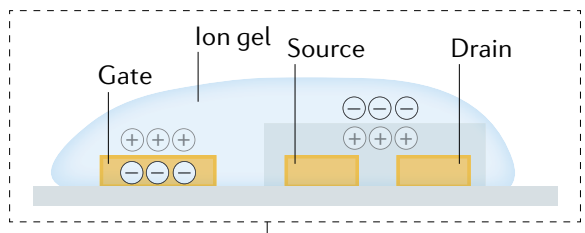

(3) Voltage pulses

$\square \square$
(4) Postsynaptic currents M 
Fig. 8 | Synaptics and neuromorphics with EGTs. a Schematic synaptic organic electrochemical transistor (OECT) in analogy with a biological synapse. $\mathbf{b}$ | Example of a pair of presynaptic pulses $\left(V_{\text {Pre }}\right)$ applied at the OECT gate electrode and the postsynaptic drain current $\left(I_{\text {Post }}\right)$ measured as a function of time. Amplitude, $A$, of $I_{\text {Post }}$ exhibits paired pulse depression behaviour. $\mathbf{c}$ | Biological afferent nerve stimulated by pressure. Action potentials from multiple nerve fibres combine through synapses and contribute to information processing. $\mathbf{d}$ |Schematic structure of an artificial afferent nerve. EGT, electrolyte-gated transistor; PEDOT:PSS, poly(3,4-ethylenedioxythiophene) polystyrene sulfonate; $V_{D S}$, drain-source voltage. Parts $\mathbf{a}$ and $\mathbf{b}$ adapted with permission from REF. ${ }^{274}$, Wiley. Parts $\mathbf{c}$ and $\mathbf{d}$ adapted with permission from REF. ${ }^{282}$, AAAS.

conductance combined with low power consumption and decoupled 'write' and 'read' operations are key advantages of EGTs compared with dielectric transistors and two-terminal devices ${ }^{267,268}$. The analogy between neurotransmitters diffusing through the synaptic cleft and ions drifting in and out of the channel of ion-permeable EGTs, such as OECTs (FIG. 8a), is an attractive approach to understand the working mechanism of OECT-based artificial synapses ${ }^{269}$. As displayed in FIG. $8 \mathrm{~b}$, the postsynaptic drain current shows paired pulse depression behaviour as a function of time $\left(I_{\text {Post }}\right)$ by applying a pair of presynaptic pulses $\left(V_{\mathrm{Pre}}\right)$ at the gate electrode of an OECT. In addition, the hysteretic behaviour of OECT electrical characteristics is due to the relatively slow kinetics of ion penetration into the polymer. This behaviour, in turn, results in an inherently volatile operation with a slow timescale. Enhanced chemical stability of the polymers in the electrolyte and reduced parasitic leakage currents through the external circuit ${ }^{270}$ are essential to improve the retention time. Using highcapacitance polarizable gate electrodes essentially transforms the OECT into a capacitor and, relying on the slow discharge of this capacitor, a volatile artificial synapse for neuromorphic computing has been demonstrated.

The memristive behaviour of the polyaniline devices was used to demonstrate XOR logic classification ${ }^{271}$, to simulate the dysfunction associated with Alzheimer disease $^{272}$ and, more recently, to couple electrically with cortical neurons ${ }^{273}$. Further developments of the use of the EGT for synaptic operations involved multiple gate or multiple channel configurations ${ }^{49,270,274,275}$. Indeed, an electrolyte common to multiple channels enables the emulation of the common electrochemical environment in which biological neural networks are typically immersed giving rise to mutual feedbacks ${ }^{45}$. In this context, the use of multiple inputs operating on a single output, or the use of a single input affecting multiple outputs, can simulate functions such as orientation selectivity ${ }^{276}$, homeoplasticity ${ }^{277}$ and coordinated voltage oscillations ${ }^{79}$.

One unique aspect of implementing artificial synapses with redox-active polymers is the ability to couple electrochemistry on biomolecules with a purely electronic function, using biocompatible materials. Dopamine secreted by cells could be used to induce long-term potentiation embodied as a non-volatile conductance modulation across a device channel ${ }^{48}$, thereby demonstrating a key synaptic functionality across the biotic/abiotic interface. Integration of neuromorphic devices with living matter is a promising research direction. Other important future areas of research involve applications where some amount of computation must be performed untethered and 'at the edge', which for instance would be the case for the sensory arm of a soft robot. The octopus provides an interesting model along these lines, as its tentacles contain a relatively large number of neurons and are thus capable of sensing, local computation and learning without involving the central brain.

Prosthetics and robotics. The EGT-based synaptic device can be applied to neuromorphic signal processing from multiple sensors, which is attractive for e-skin prosthetic and robotic applications because many sensors are required to detect the shape, size, texture and consistency of objects. In addition, immediate decisions on required actions need to be made based on detected information, especially for example a future threat. Therefore, fast processing of a large amount of data is required. Conventional data collection and processing methods scan each sensor individually to construct the information, but this process is slow and power-intensive when many sensors are involved. In biological nervous systems, synapses process information with a distributed, parallel and event-driven computation approach. For example, the tactile information in a biological somatosensory system (FIG. 8c) is processed by integrating signals from multiple tactile inputs at the synapses in order to partially process the information before delivering it to the brain ${ }^{278,279}$. To mimic slow-adapting type I mechanoreceptor responses to tactile pressure input signals, the generated spike train frequency should increase with input pressure ${ }^{280}$. Recently, by combining a resistive pressure sensor with organic transistor ring oscillators, a pressure-dependent tunable frequency - in which the higher the pressure, the higher the ring oscillator frequency - has been demonstrated and then extended to multiple pressure sensors ${ }^{281,282}$ (FIG. 8d). The outputs were further used as gate inputs of a synaptic EGT, and by properly selecting the polymer semiconductor it was possible to mimic the synapsis of biological afferent nerves ${ }^{279,283}$. This artificial afferent neuron (FIG. $8 d$ ) consumed $\sim 8 \mathrm{~mW}$ in the OFF state and $\sim 25 \mathrm{~mW}$ in the ON state ${ }^{282}$. The EGT synaptic transistor can be gated by multiple inputs coming from multiple ring oscillators and combines the information. The signals from two pressure sensors were combined in a postsynaptic current with two frequency components corresponding to the two inputs, which is analogous to the connection of postsynaptic neuron dendrites with biological synapses from multiple neurons. This approach can be applied to various types of sensors, such as temperature, strain or light ${ }^{284-286}$, as well as to mimic various sensory functions of biological systems, such as smell, taste and vision ${ }^{285-288}$.

\section{Reproducibility and data deposition}

The EGT fabrication together with any post-fabrication treatments and the bioelectronic experimental set-up should be carefully outlined to guarantee the reproducibility of the experiments. Specifically, the relevant EGT dimensions - including, for example, the channel and gate geometries, the gate to channel or the gate to bio-layer distance and the bio-layer to channel distance - should be accurately reported, with supporting metrology data. 
Gate voltage scanning

A method in which the gate voltage is swept from an initial value to a final value, typically with a constant voltage and time step size.
The thickness of the channel is very important in OECTs because they are ion-permeable volumetric devices. There is no standardization of electrolyte composition to date, but PBS is typically used for biological applications, as it has similar $\mathrm{pH}$ and ionic composition to typical biological fluids. However, operation in complex cell culture media is essential for applications with cells in vitro.

Figures of merit used for the direct comparison of EGT performance include the transconductance $g_{\mathrm{m}}$; the threshold voltage $V_{\mathrm{T}}$; the electronic mobility $\mu$, which is extracted from the transfer characteristics in the linear or saturation region; the $\mathrm{ON}$-to-OFF drain current ratio; and the gate and channel capacitances per unit area $\left(C^{\prime}\right)$ or per unit volume $\left(C^{*}\right)$. When ion-permeable EGTs based on mixed conductors are used, a relevant figure of merit is the product of the electronic mobility and volumetric charge storage capacity $\left(\mu C^{\star}\right)^{28}$. This product can guide materials design and processing, making OECTs a tool for the design and understanding of novel organic mixed conductors.

When EGTs are applied for in vitro evaluation, the characterization should include response to voltage pulse at the gate, which allows for understanding the time needed to reach steady state, and output curves where the voltage is scanned up and then down to show any hysteresis. The characterization protocol including delays between sourcing and measurement should also be carefully reported.

When EGTs are applied as biosensors, device stabilization should be provided by measuring several transfer and/or output characteristics with both forward and backward gate voltage scanning. The stabilization time as well as the various measurement parameters including the sweep rate (millivolts per second), the continuous or pulsing application of the voltages and the zero conditions applied, such as zero voltage/current or high impedance, should be reported. Negative control experiments are essential for the calculation of the limit of detection and, in general, they enable assessing the proper biosensor operation.

To date, there is no standard for data deposition, which is mainly due to the very wide range of application fields covered by EGTs. Standards for data deposition could help sharing and verification of the results obtained from various research groups and could help speed up the reproducibility of the results among different laboratories.

\section{Limitations and optimizations}

Ensuring high stability of operation is essential to design enhanced EGT-based bioelectronics applications beyond optimization of the EGT figures for the target application. Ultimately, EGT stability is the pre-requisite for any bioelectronic application, especially when large amplification is achieved, because EGT instabilities can also be amplified. By way of example, the variation of EGT characteristics during electrophysiology measurements could result in artefacts on the recorded signal; instabilities of EGT-based biosensors worsen the limit of detection; and stable operation is critical for cell monitoring where the EGT is exposed for days or weeks to the cultured media environment and leaching of the device materials could take place ${ }^{117,226}$. As a consequence, specific control experiments should always be run before starting the biological measurements. Although leaching is an extreme situation, the most common sources of EGT instabilities include the electrochemical redox reactions between the EGT materials, such as those used for the gate, channel, source and drain, and the electrolyte or the biolayer embedded in the EGT; morphological changes of the channel material due to hydrophobic-hydrophilic interactions with the electrolyte; and water diffusion and ion doping during long-term operation ${ }^{289,290}$. EGT degradation can also take place in ambient conditions $s^{117,134,291-297}$. This path of degradation could be a result of morphological and/or chemical interactions on the surface semiconductors or in the whole bulk of the EGT channel ${ }^{116,117,291,296,298}$. For example, a recent study investigated the stability of water-gated EGTs based on the widely used and commercially available P3HT semiconductor ${ }^{291}$. The stability was analysed by means of atomic force microscopy, X-ray photoelectron spectroscopy, electrical measurements and modelling, continuously operating the EGTs for 1 week in water. The work showed that once operational stability is achieved, dry conditions must be avoided. A gel matrix keeping the semiconducting channel hydrated was proposed, demonstrating a shelf-life of up to 2 months. In general, the degradation phenomena depend on the specific materials used for the EGT and, considering the large palette of materials, further extensive work is required in this direction. Optimization of stability in the operational environment and of the shelf-life in ambient conditions is essential for the practical application and commercialization of EGTs.

Particular attention to potential faradaic side reactions during the EGT operation is important as reactive side products may accelerate the device degradation and may also be harmful for the local biological environment. To date, an increasing number of efforts are devoted to improving EGT performance and stability, including applied voltages under quasi-static and dynamic operation $^{153,291}$, physical-based models ${ }^{23,299}$, improvement of the device architecture $\mathrm{e}^{150,300-302}$ and new materials specifically designed for reducing the redox reactions and improving the ionic-electronic coupling $29,30,32,111,115,303$.

Gate functionalization strategies. The main limitations of functionalizing the gate are the limited coverage, low density and compactness of the bio-recognition layer. The non-specific adsorption impacts the sensitivity and robustness of the sensor parameters. Direct immobilization of the bio-recognition moiety bearing a thiolated linker is straightforward, but might lead to protein denaturation. Immobilization via solvent-exposed cysteines might result in uncontrolled orientation if several cysteines are amenable for surface binding, which is, however, a rare occurrence.

As an alternative to the strategies detailed above, the bio-recognition unit can be immobilized at the channel surface $^{169,304,305}$. This approach requires derivatization of semiconductor molecules and might be challenging. Moreover, it could result in a lowering of the surface 


\section{Plasma}

A gas of ions with one or more orbital electrons stripped and free electrons electronic mobility of the semiconductor and this limitation could be overcome by the gate bio-functionalization approach. Nevertheless, immobilizing recognition elements onto, or within, the channel of EGT biosensors has been reported. Examples include phospholipid bilayer membranes with embedded proteins assembled on the semiconductor channel ${ }^{200}$; plasma-enhanced chemical vapour deposition of hydrophilic coatings ${ }^{306}$; P3HT with selective cation-binding pending groups ${ }^{307}$, or with biotin ${ }^{308}$; and biotin grafted onto the bottom channel dielectric beneath the semiconductor channel ${ }^{309}$. A recent example envisions self-organization of the semiconductor materials (single-walled carbon nanotube transistors) with a protein that acts both as a surfactant for processing and as a bio-recognition element. Along this direction, cell membranes have more recently also been used to functionalize channel sensors to monitor the activity of transmembrane proteins such as ion channels ${ }^{310,311}$. This framework, based on hybrid bio-organic nanomaterials, could be extended to a larger number of relevant cases.

Although organic electronics relies on extensive materials libraries for applications such as organic light emitting diodes and organic photovoltaics, the number of active materials used in organic bioelectronics is very limited to date and materials tailored for bioelectronics applications are lacking. For developing the field, we believe that an effort in endowing OSCs with bio-recognition and other biological functions, for instance biocompatibility, is important.

\section{Outlook}

EGTs are ideally suited to be interfaced with functional biosystems - the latter being a film of biological recognition elements, a layer of cells, a tissue or, even, an organ. EGTs can operate in the same liquid environment that ensures the physiological functioning of the biological entity to whom they are interfaced $^{15,117,147,191,202,208,215,216,294,303}$. Moreover, the ability of EGTs to operate directly in an electrolyte as well as amplify and transduce both ionic and electronic currents enables a unique and convenient communication platform within biology.

These specific properties are connected with the development and optimization of all of the EGT components, from the channel materials to the device structures. With respect to the materials used, various classes of OSCs including CPEs, conjugated polymers and composites have been developed. Inorganic counterparts encompass amorphous structures of indium-galliumzinc oxide as well as two-dimensional materials such as graphene and molybdenum disulfide. Device structures range from including a convenient side or coplanar gate to a floating gate-based architecture, which can be fabricated by photolithographic techniques and additive printing processes. Invariably, the interface with the biosystem is present, which is deposited onto one of the EGT electronic interfaces. Selectivity to specific biological events can be obtained by grafting recognition elements on the EGT surface or surfaces. Modelling device activities and their key functional parameters can contribute to improve control over the EGT's performance level. To this end, various equivalent circuits including the bio-layer residing at one of the transistor electronic interfaces is accounted for.

EGTs integrating or interfacing a biosystem enable key applications that revolutionize how health care is provided. For example, the application of EGTs to in vivo electrophysiology holds significant promise. EGTs are suitable for electrophysiology recordings and enable transducer miniaturization, but there is still room for improved solutions on this front. With respect to studying cultured cells, a dimensional transformation in cell biology is underway, adapting two-dimensional methods to growing cells in three dimensions to better reflect the real environment of cells. The EGT electronic control of such systems is showing a great deal of potential, such as in the case of the three-dimensional cultures of spheroids. The use of EGTs as extremely sensitive and selective biosensors is an exciting area, where a large-area EGT has been shown to detect down to a few biomarkers. Such an assay is label-free and can be carried out in a few minutes directly in a non-pretreated real sample. EGT bioelectronic sensors can revolutionize our current approach to point-of-care devices, which are essential for early diagnosis of diseases as well as bacterial or viral infections. Further, applying EGTs to synaptic and neuromorphic bio-interfaces has enabled the development of neuromorphic EGTs, which provide large and tunable ionic-electronic conductance, low power consumption and decoupled 'write' and 'read' operations. Important future areas of research involve applications where EGTs can simulate the dysfunction associated with Alzheimer disease, and can couple electrically with cortical neurons. Further, EGT-based synaptic signal processing from multiple sensors can find applications for e-skin prosthetics and robotics, where several sensors are required to detect the shape, size, texture and consistency of objects. However, a very rapid decision on required actions needs to be made based on detected information, especially in case of a future hazard.

Stability of the device operation, shortcomings of gate functionalization strategies and development of standard characterization and measurement protocols are important and require further improvements. Finally, it is very important to continue gathering insights into the device functional mechanisms at all levels, from the materials involved to the device detecting mechanisms and the behaviour of the bio-layer or the bio-interface during device operation.

Published online: 07 October 2021
1. Willner, I. \& Katz, E. (eds) Bioelectronics: From Theory to Applications (Wiley, 2005)

2. Hess, L. H., Seifert, M. \& Garrido, J. A. Graphene transistors for bioelectronics. Proc. IEEE 101 1780-1792 (2013)

3. Zhou, W., Dai, X. \& Lieber, C. M. Advances in nanowire bioelectronics. Rep. Prog. Phys. 80, 16701 (2016).
4. Rivnay, J. et al. Organic electrochemical transistors Nat. Rev. Mater. 3, 17086 (2018). This review provides an overview of OECTs. 5. Wang, N., Yang, A., Fu, Y., Li, Y. \& Yan, F. Functionalized organic thin film transistors for biosensing. Acc. Chem. Res. 52, 277-287 (2019).
6. Kim, S. H. et al. Electrolyte-gated transistors for organic and printed electronics. Adv. Mater. 25, 1822-1846 (2013).

7. Bisri, S. Z., Shimizu, S., Nakano, M. \& Iwasa, Y. Endeavor of iontronics: from fundamentals to applications of ion-controlled electronics. Adv. Mater. 29, 1-48 (2017). 
8. Jiang, J., Wan, Q., Sun, J. \& Lu, A. Ultralow-voltage transparent electric-double-layer thin-film transistors processed at room-temperature. Appl. Phys. Lett. 95, 3-6 (2009).

9. Chae, M. S., Park, J. H., Son, H. W., Hwang, K. S. \& Kim, T. G. IGZO-based electrolyte-gated field-effect transistor for in situ biological sensing platform. Sensors Actuators. B Chem. 262, 876-883 (2018)

10. Woo Son, H., Park, J. H., Chae, M.-S., Kim, B.-H. \& Kim, T. G. Bilayer indium gallium zinc oxide electrolytegated field-effect transistor for biosensor platform with high reliability. Sens. Actuators B Chem. 312, 127955 (2020).

11. Zhu, J. et al. Ion gated synaptic transistors based on 2D van der Waals crystals with tunable diffusive dynamics. Adv. Mater. 30, 1800195 (2018).

12. Song J., Chu, Y, Liu, Z \& Xu, H. Fabrication of solution-processed ambipolar electrolyte-gated field effect transistors from $\mathrm{a} \mathrm{MoS}_{2}$-polymer hybrid for multifunctional optoelectronics. J. Mater. Chem. C. 9, 1701-1708 (2021)

13. Wan, C. J. et al. Proton-conducting graphene oxidecoupled neuron transistors for brain-inspired cognitive systems. Adv. Mater. 28, 3557-3563 (2016)

14. Furlan de Oliveira, R. et al. Liquid-gated transistors based on reduced graphene oxide for flexible and wearable electronics. Adv. Funct. Mater. 29, 1905375 (2019).

15. Joshi, S. et al. Ambient processed, water-stable, aqueous-gated sub $1 \mathrm{~V}$ n-type carbon nanotube field effect transistor. Sci. Rep. 8, 11386 (2018).

16. Cho, K. G. et al. Printable carbon nanotube-based elastic conductors for fully-printed sub- $1 \mathrm{~V}$ stretchable electrolyte-gated transistors and inverters. J. Mater Chem. C. 8, 3639-3645 (2020).

17. Lu, S. \& Franklin, A. D. Printed carbon nanotube thin-film transistors: progress on printable materials and the path to applications. Nanoscale 12 , 23371-23390 (2020).

18. Cho, J. H. et al. Printable ion-gel gate dielectrics for low-voltage polymer thin-film transistors on plastic. Nat. Mater. 7, 900-906 (2008). This article describes printable ion-gel gated polymer transistors.

19. Leonardi, F. et al. Electrolyte-gated organic field-effect transistor based on a solution sheared organic semiconductor blend. Adv. Mater. 28, 10311-10316 (2016).

20. Zeglio, E. \& Inganäs, O. Active materials for organic electrochemical transistors. Adv. Mater. 30, 180094 (2018).

21. Proctor, C. M., Rivnay, J. \& Malliaras, G. G. Understanding volumetric capacitance in conducting polymers. J. Polym. Sci. B Polym. Phys. 54 , 1433-1436 (2016)

22. Volkov, A. V. et al. Understanding the capacitance of PEDOT:PSS. Adv. Funct. Mater 27, 1700329 (2017).

23. Tybrandt, K., Zozoulenko, I. V. \& Berggren, M Chemical potential-electric double layer coupling in conjugated polymer-polyelectrolyte blends. Sci. Ad https://doi.org/10.1126/sciadv.aao3659 (2017).

24 Romele, P., Ghittorelli, M., Kovăcs-Vajna, Z. M. \& Torricelli, F. Ion buffering and interface charge enable high performance electronics with organic electrochemical transistors. Nat. Commun. 10, 3044 (2019).

25. Moser, M., Ponder, J. F. Jr, Wadsworth, A Giovannitti, A. \& McCulloch, I. Materials in organic electrochemical transistors for bioelectronic applications: past, present, and future. Adv. Funct Mater. 29, 1807033 (2019).

26. Borges-González, J., Kousseff, C. J. \& Nielsen, C. B. Organic semiconductors for biological sensing. J. Mater. Chem. C. 7, 1111-1130 (2019).

27. Paulsen, B. D., Tybrandt, K., Stavrinidou, E. \& Rivnay, J. Organic mixed ionic-electronic conductors. Nat. Mater. 19, 13-26 (2020).

This article presents an insightful review of organic mixed ionic-electronic conductors.

28. Inal, S., Malliaras, G. G. \& Rivnay, J. Benchmarking organic mixed conductors for transistors. Nat Commun. 8, 1767 (2017).

29. Sun, H., Gerasimov, J., Berggren, M. \& Fabiano, S n-Type organic electrochemical transistors: materials and challenges. J. Mater. Chem. C. 6, 11778-11784 (2018).

30. Giovannitti, A. et al. n-Type organic electrochemical transistors with stability in water. Nat. Commun. 7 . 13066 (2016)

31. Lill, A. T. et al. Organic electrochemical transistors based on the conjugated polyelectrolyte PCPDTBT-SO 3K (CPE-K). Adv. Mater. 32, 1908120 (2020).
32. Parr, Z. S. et al. Semiconducting small molecules as active materials for $p$-type accumulation mode organic electrochemical transistors. Adv. Electron. Mater. 6 2000215 (2020).

33. White, H. S., Kittlesen, G. P. \& Wrighton, M. S. Chemical derivatization of an array of three gold microelectrodes with polypyrrole: fabrication of a molecule-based transistor. J. Am. Chem. Soc. 106, 5375-5377 (1984).

34. Bergveld, P. Development of an ion-sensitive solidstate device for neurophysiological measurements. IEEE Trans. Biomed Eng. BME-17, 70-71 (1970).

35. Bergveld, P. Development, operation, and application of the ion-sensitive field-effect transistor as a tool for electrophysiology. IEEE Trans. Biomed. Eng. BME-19, 342-351 (1972)

36. Matsuo, T. \& Wise, K. D. An integrated field-effect electrode for biopotential recording. IEEE Trans. Biomed. Eng. BME-21, 485-487 (1974).

37. Janata, J. Historical review: twenty years of ion-selective field-effect transistors. Analyst 119 , 2275-2278 (1994)

38. Moser, N., Lande, T. S., Toumazou, C. \& Georgiou, P. ISFETs in CMOS and emergent trends in instrumentation: a review. IEEE Sens. J. 16 6496-6514 (2016)

39. Bai, L. et al. Biological applications of organic electrochemical transistors: electrochemical biosensors and electrophysiology recording. Front. Chem. 7, 313 (2019).

40. Masvidal-Codina, E. et al. High-resolution mapping of infraslow cortical brain activity enabled by graphene microtransistors. Nat. Mater. 18, 280-288 (2019).

41. Kyndiah, A. et al. Bioelectronic recordings of cardiomyocytes with accumulation mode electrolyte gated organic field effect transistors. Biosens. Bioelectron. 150, 111844 (2020).

42. Schmoltner, K., Kofler, J., Klug, A. \& List-Kratochvil, E. J. W. Electrolyte-gated organic field-effect transistor for selective reversible ion detection Adv Mater 25, 6895-6899 (2013).

43. Sessolo, M., Rivnay, J., Bandiello, E., Malliaras, G. G. \& Bolink, H. J. Ion-selective organic electrochemical transistors. Adv. Mater. 26, 4803-4807 (2014).

44. Melzer, K. et al. Selective ion-sensing with membranefunctionalized electrolyte-gated carbon nanotube fieldeffect transistors. Analyst 139, 4947-4954 (2014).

45. Gkoupidenis, P., Koutsouras, D. A. \& Malliaras, G. G. Neuromorphic device architectures with global connectivity through electrolyte gating. Nat. Commun 8, 15448 (2017).

This article is the first demonstration of electrolyte-gated neuromorphic devices with global connectivity.

46. Jiang, J. et al. 2D MoS 2 neuromorphic devices for brain-like computational systems. Small 13, 1700933 (2017).

47. Gerasimov, J. Y et al. An evolvable organic electrochemical transistor for neuromorphic applications. Adv. Sci. 6, 1801339 (2019)

48. Keene, S. T. et al. A biohybrid synapse with neurotransmitter-mediated plasticity. Nat. Mater. 19, 969-973 (2020)

This article is the first demonstration of a biohybrid synapse with neurotransmitter-mediated plasticity.

49. Ling, H. et al. Electrolyte-gated transistors for synaptic electronics, neuromorphic computing and adaptable biointerfacing. Appl. Phys. Rev. 7, 011307 (2020).

50. Berto, M. et al. EGOFET peptide aptasensor for label-free detection of inflammatory cytokines in complex fluids. Adv. Biosyst. 2, 1700072 (2018)

51. Macchia, E. et al. Label-free and selective singlemolecule bioelectronic sensing with a millimeter-wide self-assembled monolayer of anti-immunoglobulins. Chem. Mater. 31, 6476-6483 (2019)

52. Ricci, S. et al. Label-free immunodetection of $\alpha$-synuclein by using a microfluidics coplanar electrolyte-gated organic field-effect transistor. Biosens. Bioelectron. 167, 112433 (2020)

53. Macchia, E. et al. Ultra-low HIV-1 p24 detection limits with a bioelectronic sensor. Anal. Bioanal. Chem 412, 811-818 (2020)

54. Pappa, A.-M. et al. Organic transistor arrays integrated with finger-powered microfluidics for multianalyte saliva testing. Adv. Healthc. Mater. $\mathbf{5}$, 2295-2302 (2016).

55. Fenoy, G. E., Marmisollé, W. A., Azzaroni, O. $\&$ Knoll, W. Acetylcholine biosensor based on the electrochemical functionalization of graphene field-effect transistors. Biosens. Bioelectron. 148 111796 (2020).
56. Ohayon, D. et al. Biofuel powered glucose detection in bodily fluids with an n-type conjugated polymer. Nat. Mater. 19, 456-463 (2020).

57. Hajian, R. et al. Detection of unamplified target genes via CRISPR-Cas9 immobilized on a graphene field-effect transistor. Nat. Biomed. Eng. 3, 427-437 (2019).

58. Campos, R. et al. Attomolar label-free detection of DNA hybridization with electrolyte-gated graphene field-effect transistors. ACS Sens. 4, 286-293 (2019).

59. Hwang, M T et al. Ultrasensitive detection of nucleic acids using deformed graphene channel field effect biosensors. Nat. Commun. 11, 1543 (2020).

60. Zhang, Z., Zheng, T. \& Zhu, R. Long-term and label-free monitoring for osteogenic differentiation of mesenchymal stem cells using force sensor and impedance measurement. J. Mater. Chem. B 8, 9913-9920 (2020).

61. Decataldo, F. et al. Organic electrochemical transistors for real-time monitoring of in vitro silver nanoparticle toxicity. Adv. Biosyst. 4, 1900204 (2020).

62. Sun, $C$. et al. Facile and cost-effective liver cancer diagnosis by water-gated organic field-effect transistors. Biosens. Bioelectron. 164, 11225 (2020).

63. Danielson, E. et al. Non-enzymatic and highly sensitive lactose detection utilizing graphene field-effect transistors. Biosens. Bioelectron. 165, 112419 (2020).

64. Kanai, Y. et al. Graphene field effect transistor-based immunosensor for ultrasensitive noncompetitive detection of small antigens. ACS Sens. 5, 24-28 (2020).

65. Wu, D. et al. Dual-aptamer modified graphene fieldeffect transistor nanosensor for label-free and specific detection of hepatocellular carcinoma-derived microvesicles. Anal. Chem. 92, 4006-4015 (2020).

66. Seo, G. et al. Rapid detection of COVID-19 causative virus (SARS-CoV-2) in human nasopharyngeal swab specimens using field-effect transistor-based biosensor. ACS Nano 14, 5135-5142 (2020).

67. Jimison, L. H. et al. Measurement of barrier tissue integrity with an organic electrochemical transistor. Adv. Mater. 24, 5919-5923 (2012).

68. Ferro, M. P. et al. Effect of e cigarette emissions on tracheal cells monitored at the air-liquid interface using an organic electrochemical transistor. Adv. Biosyst. 3, 1800249 (2019).

69. Lingstedt, L. V. et al. Monitoring of cell layer integrity with a current-driven organic electrochemical transistor. Adv. Healthc. Mater. 8, 1900128 (2019).

70. Pierre, A., Doris, S. E., Lujan, R. \& Street, R. A Monolithic integration of ion-selective organic electrochemical transistors with thin film transistors on flexible substrates. Adv. Mater. Technol. 4 1800577 (2019).

71. Demuru, S., Kunnel, B. P. \& Briand, D. Real-time multi-ion detection in the sweat concentration range enabled by flexible, printed, and microfluidicsintegrated organic transistor arrays. Adv. Mater. Technol. 5, 2000328 (2020).

72. Han, S., Yamamoto, S., Polyravas, A. G. \& Malliaras, G. G. Microfabricated ion-selective transistors with fast and super-Nernstian response. Adv. Mater. 32, 2004790 (2020).

73. Romele, P. et al. Multiscale real time and high sensitivity ion detection with complementary organic electrochemical transistors amplifier. Nat Commun. 11, 1-11 (2020) This article is the first demonstration of local detection and amplification with an iontronic integrated amplifier for multifunctional bioelectronics.

74. Spyropoulos, G. D., Gelinas, J. N. \& Khodagholy, D. Internal ion-gated organic electrochemical transistor: a building block for integrated bioelectronics. Sci. Adv . 5, eaau7378 (2019).

75. Cea, C. et al. Enhancement-mode ion-based transistor as a comprehensive interface and real-time processing unit for in vivo electrophysiology. Nat. Mater. 19 679-686 (2020)

76. Gualandi, I. et al. Textile organic electrochemical transistors as a platform for wearable biosensors. Sci. Rep. 6, 33637 (2016).

77. Sensi, M. et al. Modulating the faradic operation of all-printed organic electrochemical transistors by facile in situ modification of the gate electrode. ACS Omega 4, 5374-5381 (2019)

78. Minamiki, T., Hashima, Y., Sasaki, Y. \& Minami, T. An electrolyte-gated polythiophene transistor for the detection of biogenic amines in water. Chem. Commun. 54, 6907-6910 (2018) 
79. Koutsouras, D. A., Prodromakis, T., Malliaras, G. G., Blom, P. W. M. \& Gkoupidenis, P. Functional connectivity of organic neuromorphic devices by global voltage oscillations. Adv. Intell. Syst. 1, 1900013 (2019).

80. White, S. P., Dorfman, K. D. \& Frisbie, C. D. Operating and sensing mechanism of electrolyte-gated transistors with floating gates: building a platform for amplified biodetection. J. Phys. Chem. C. 120 108-117 (2016)

81. White, S. P., Sreevatsan, S., Frisbie, C. D. \& Dorfman, K. D. Rapid, selective, label-free aptameric capture and detection of ricin in potable liquids using a printed floating gate transistor. ACS Sens. 1 , 1213-1216 (2016)

82. White, S. P., Dorfman, K. D. \& Frisbie, C. D. Label-free DNA sensing platform with low-voltage electrolytegated transistors. Anal. Chem. 87, 1861-1866 gated transi
(2015).

83. White, S. P., Frisbie, C. D. \& Dorfman, K. D. Detection and sourcing of gluten in grain with multiple floating gate transistor biosensors. ACS Sens. 3, 395-402 (2018).

84. Lai, S., Barbaro, M. \& Bonfiglio, A. Tailoring the sensing performances of an OFET-based biosensor. Sens. Actuators B Chem. 233, 314-319 (2016).

85. Spanu, A. et al. An organic transistor-based system for reference-less electrophysiological monitoring of excitable cells. Sci. Rep. 5, 1-7 (2015).

86. Leighton, C. Electrolyte-based ionic control of functional oxides. Nat. Mater. 18, 13-18 (2019).

87. Xu, K. \& Fullerton-Shirey, S. K. Electric-double-layergated transistors based on two-dimensional crystals: recent approaches and advances. J. Phys. Mater. 3, 032001 (2020).

88. Hai, Z., Wei, Z., Xue, C., Xu, H. \& Verpoort, F. Nanostructured tungsten oxide thin film devices: from optoelectronics and ionics to iontronics. J. Mater. Chem. C. 7, 12968-12990 (2019).

89. Fan, Q. et al. Solution-gated transistors of twodimensional materials for chemical and biological sensors: status and challenges. Nanoscale 12, 11364-11394 (2020).

90. Manoli, K. et al. Printable bioelectronics to investigate functional biological interfaces. Angew. Chem. Int. Ed. 54, 12562-12576 (2015).

91. Picca, R. A. et al. Ultimately sensitive organic bioelectronic transistor sensors by materials and device structure design. Adv. Funct. Mater. 30, 1-23 (2020).

92. He, Y., Yang, Y., Nie, S., Liu, R. \& Wan, Q Electric-double-layer transistors for synaptic devices and neuromorphic systems. J. Mater. Chem. C. 6 , 5336-5352 (2018)

93. Bao, C. \& Kim, W. S. Perspective of printed solid-state ion sensors toward high sensitivity and selectivity. Adv. Eng. Mater. 22, 1-14 (2020).

94. Liu, J., Zhao, F., Li, H. \& Pei, Q. Electrolyte-gated light-emitting transistors: working principle and applications. Mater. Chem. Front. 2, 253-263 (2018).

95. Zhu, J., Zhang, T., Yang, Y. \& Huang, R. A comprehensive review on emerging artificial neuromorphic devices. Appl. Phys. Rev. 7, 011312 (2020).

96. Jiang, S. et al. Emerging synaptic devices: from two-terminal memristors to multiterminal neuromorphic transistors. Mater. Today Nano 8 , 101846 (2019)

97. Zeglio, E. et al. Conjugated polyelectrolyte blends for electrochromic and electrochemical transistor devices. Chem. Mater. 27, 6385-6393 (2015).

98. Rivnay, J. et al. Structural control of mixed ionic and electronic transport in conducting polymers. Nat. Commun. 7, 11287 (2016).

99. Kim, S.-M. et al. Influence of PEDOT:PSS crystallinity and composition on electrochemical transistor performance and long-term stability. Nat. Commun. 9 3858 (2018)

100. Kim, Y. et al. Strain-engineering induced anisotropic crystallite orientation and maximized carrier mobility for high-performance microfiber-based organic bioelectronic devices. Adv Mater 33, 2007550 (2021).

101. Inal, S. et al. Organic electrochemical transistors based on PEDOT with different anionic polyelectrolyte dopants. J. Polym. Sci. B Polym. Phys. 54, 147-151 (2016).

102. Singh, T. B. et al. High-performance ambipolar pentacene organic field-effect transistors on poly(vinyl alcohol) organic gate dielectric. Adv. Mater. 17, 2315-2320 (2005)
103. Yang, S. Y., Shin, K. \& Park, C. E. The effect of gatedielectric surface energy on pentacene morphology and organic field-effect transistor characteristics. Adv. Funct. Mater. 15, 1806-1814 (2005).

104. Cramer, T. et al. Double layer capacitance measured by organic field effect transistor operated in water. Appl. Phys. Lett. 100, 143302 (2012).

105. Chang, J.-F. et al. Enhanced mobility of poly(3-hexylthiophene) transistors by spin-coating from high-boiling-point solvents. Chem. Mater. 16, 4772-4776 (2004).

106. Kergoat, L. et al. A water-gate organic field-effect transistor. Adv. Mater. 22, 2565-2569 (2010)

107. Mulla, M. Y. et al. Capacitance-modulated transisto detects odorant binding protein chiral interactions. Nat. Commun. 6, 6010 (2015).

108. Li, J. et al. A stable solution-processed polymer semiconductor with record high-mobility for printed transistors. Sci. Rep. 2, 754 (2012)

109. Nguyen, T. T. K. et al. Triggering the electrolyte-gated organic field-effect transistor output characteristics through gate functionalization using diazonium chemistry: application to biodetection of 2,4-dichlorophenoxyacetic acid. Biosens. Bioelectron. 113, 32-38 (2018)

110. Bronstein, H., Nielsen, C. B., Schroeder, B. C. \& McCulloch, I. The role of chemical design in the performance of organic semiconductors. Nat. Rev. Chem. 4, 66-77 (2020)

111. Moser, M. et al. Side chain redistribution as a strategy to boost organic electrochemical transistor performance and stability. Adv. Mater. 32, 1-6 (2020).

112. Moser, M. et al. Polaron delocalization in donoracceptor polymers and its impact on organic electrochemical transistor performance. Angew. Chem. Int. Ed. 60, 7777-7785 (2021).

113. Moser, M. et al. Controlling electrochemically induced volume changes in conjugated polymers by chemical design: from theory to devices. Adv. Funct. Mater. 31 2100723 (2021)

114. Sun, H. et al. Complementary logic circuits based on high-performance $n$-type organic electrochemical transistors. Adv. Mater. 30, 1704916 (2018)

115. Giovannitti, A. et al. Energetic control of redox-active polymers toward safe organic bioelectronic materials. Adv. Mater. 32, 1908047 (2020)

116. Flagg, L. Q. et al. Polymer crystallinity controls water uptake in glycol side-chain polymer organic electrochemical transistors. J. Am. Chem. Soc. 141, 4345-4354 (2019).

117. Zhang, Y., Zeng, Q., Shen, Y., Yang, L. \& Yu, F. Electrochemical stability investigations and drug toxicity tests of electrolyte-gated organic field-effect transistors. ACS Appl. Mater. Interfaces 12 , 56216-56221 (2020).

118. Maria, I. P. et al. The effect of alkyl spacers on the mixed ionic-electronic conduction properties of n-type polymers. Adv. Funct. Mater. 31, 2008718 (2021).

119. Chen, X. et al. $\mathrm{n}$-Type rigid semiconducting polymers bearing oligo(ethylene glycol) side chains for highperformance organic electrochemical transistors. Angew. Chem. Int. Ed. 60, 9368 (2021).

120. Moser, M. et al. Ethylene glycol-based side chain length engineering in polythiophenes and its impact on organic electrochemical transistor performance. Chem. Mater. 32, 6618-6628 (2020).

121. Nielsen, C. B. et al. Molecular design of semiconducting polymers for high-performance organic electrochemical transistors. J. Am. Chem. Soc 138, 10252-10259 (2016).

122. Kumar, N., Kumar, J. \& Panda, S. Back-channel electrolyte-gated a-ICZO dual-gate thin-film transistor for enhancement of $\mathrm{pH}$ sensitivity over nernst limit. IEEE Electron. Device Lett. 37, 500-503 (2016).

123. Chen, S. H. et al. High performance electric-doublelayer amorphous ICZO thin-film transistors gated with hydrated bovine serum albumin protein. Org. Electron. 24, 200-204 (2015).

124. Samanta, C., Ghimire, R. R. \& Ghosh, B. Fabrication of amorphous indium-gallium-zinc-oxide thin-film transistor on flexible substrate using a polymer electrolyte as gate dielectric. IEEE Trans. Electron. Devices 65, 2827-2832 (2018).

125. Bandiello, E., Sessolo, M. \& Bolink, H. J. Aqueous electrolyte-gated $\mathrm{ZnO}$ transistors for environmental and biological sensing. J. Mater. Chem. C. 2 , 10277-10281 (2014).

126. Hong, K., Kim, S. H., Lee, K. H. \& Frisbie, C. D. Printed, sub-2 $\mathrm{V} \mathrm{ZnO}$ electrolyte gated transistors and inverters on plastic. Adv. Mater. 25, 3413-3418 (2013).
127. Zare Bidoky, F. et al. Sub-3 V ZnO electrolyte-gated transistors and circuits with screen-printed and photocrosslinked ion gel gate dielectrics: new routes to improved performance. Adv. Funct. Mater. 30, 1902028 (2020).

128. Al Naim, A. \& Grell, M. Electron transporting watergated thin film transistors. Appl. Phys. Lett. 101 141603 (2012).

129. Dasgupta, S., Kruk, R. \& Hahn, H. Inkjet printed, high mobility inorganic-oxide field effect temperature. ACS Nano 5, 9628-9638 (2011).

130. Kim, J. et al. Fabrication of high-performance ultrathin $\mathrm{In}_{2} \mathrm{O}_{3}$ film field-effect transistors and biosensors using chemical lift-off lithography. ACS Nano 9, 4572-4582 (2015).

131. Nakatsuka, N. et al. Aptamer-field-effect transistors overcome Debye length limitations for small-molecule sensing. Science 362, 319-324 (2018).

132. Feng, X. et al. Impact of intrinsic capacitances on the dynamic performance of printed electrolyte-gated inorganic field effect transistors. IEEE Trans. Electron. Devices 66, 3365-3370 (2019).

133. Marques, G. C. et al. Influence of humidity on the performance of composite polymer electrolyte-gated field-effect transistors and circuits. IEEE Trans. Electron. Devices 66, 2202-2207 (2019).

134. Cherukupally, N., Divya, M. \& Dasgupta, S. A comparative study on printable solid electrolytes toward ultrahigh current and environmentally stable thin film transistors. Adv. Electron. Mater. 6, 2000788 (2020).

135. Ohno, Y., Maehashi, K., Yamashiro, Y. \& Matsumoto, K. Electrolyte-gated graphene field-effect transistors for detecting $\mathrm{ph}$ and protein adsorption. Nano Lett. $\mathbf{9}$, 3318-3322 (2009).

136. Hao, Z. et al. Real-time monitoring of insulin using a graphene field-effect transistor aptameric nanosensor. ACS Appl. Mater. Interfaces 9, 27504-27511 (2017).

137. Han, D., Chand, R. \& Kim, Y. S. Microscale loopmediated isothermal amplification of viral DNA with real-time monitoring on solution-gated graphene FET microchip. Biosens. Bioelectron. 93, 220-225 (2017).

138. Liu, S. et al. Detection of bisphenol a using DNA-functionalized graphene field effect transistors integrated in microfluidic systems. ACS Appl. Mater. Interfaces 10, 23522-23528 (2018)

139. Sarkar, D. et al. $\mathrm{MoS}_{2}$ field-effect transistor for next-generation label-free biosensors. ACS Nano 8 3992-4003 (2014).

140. Zhou, G. et al. Ultrasensitive mercury ion detection using DNA-functionalized molybdenum disulfide nanosheet/gold nanoparticle hybrid field-effect transistor device. ACS Sens. 1, 295-302 (2016)

141. Kelly, A. G. et al. All-printed thin-film transistors from networks of liquid-exfoliated nanosheets. Science 356, 69-73 (2017).

142. Masurkar, N., Kumar, N., Yurgelevic, S. \& Varma, S. Reliable and highly sensitive biosensor from suspended $\mathrm{MoS}_{2}$ atomic layer on nano-gap electrodes. Biosensors 172, 112724 (2020).

143. Higgins, T. M. et al. Electrolyte-gated n-type transistors produced from aqueous inks of WS nanosheets. Adv. Funct. Mater. 29, 1804387 (2019).

144. Heller, I. et al. Influence of electrolyte composition on liquid-gated carbon nanotube and graphene transistors. J. Am. Chem. Soc. 132, 17149-17156 (2010).

145. Rosenblatt, S. et al. High performance electrolyte gated carbon nanotube transistors. Nano Lett. 2, 869-872 (2002).

146. Chen, L. et al. Organic electrochemical transistors for the detection of cell surface glycans. ACS Appl. Mater. Interfaces 10, 18470-18477 (2018)

147. Scuratti, F. et al. Real-time monitoring of cellular cultures with electrolyte-gated carbon nanotube transistors. ACS Appl. Mater. Interfaces 11, 37966-37972 (2019)

148. Singaraju, S. A. et al. Development of fully printed electrolyte-gated oxide transistors using graphene passive structures. ACS Appl. Electron. Mater. 1 1538-1544 (2019).

149. Li, H. et al. Polyfluorinated electrolyte for fully printed carbon nanotube electronics. Adv. Funct. Mater. 26 6914-6920 (2016).

150. Cardenas, J. A., Lu, S., Williams, N. X., Doherty, J. L. \& Franklin, A. D. In-place printing of flexible electrolytegated carbon nanotube transistors with enhanced stability. IEEE Electron. Device Lett. 42, 367-370 (2021). 
151. Hyun, W. J., Secor, E. B., Hersam, M. C., Frisbie, C. D. \& Francis, L. F. High-resolution patterning of graphene by screen printing with a silicon stencil for highly flexible printed electronics. Adv. Mater. 27, 109-115 (2015)

152. Kim, S. H., Hong, K., Lee, K. H. \& Frisbie, C. D. Performance and stability of aerosol-jet-printed electrolyte-gated transistors based on poly(3hexylthiophene). ACS Appl. Mater. Interfaces 5 6580-6585 (2013)

153. Blasi, D. et al. Printed, cost-effective and stable poly(3-hexylthiophene) electrolyte-gated field-effect transistors. J. Mater. Chem. C. 8, 15312 (2020).

154. Scheiblin, G. et al. Screen-printed organic electrochemical transistors for metabolite sensing MRS Commun. 5, 507-511 (2015).

155. Galliani, M. et al. Flexible printed organic electrochemical transistors for the detection of uric acid in artificial wound exudate. Adv. Mater. Interfaces 7, 2001218 (2020)

156. Zabihipour, M. et al. High yield manufacturing of fully screen-printed organic electrochemical transistors. NPJ Flex. Electron. 4, 1-8 (2020)

157. Medina-Sánchez, M., Martínez-Domingo, C., Ramon, E. \& Merkoçi, A. An inkjet-printed field-effect transistor for label-free biosensing. Adv. Funct. Mater. 24, 6291-6302 (2014)

158. Bihar, E. et al. A disposable paper breathalyzer with an alcohol sensing organic electrochemical transistor. Sci. Rep. 6, 27582 (2016)

159. Cho, N. B., Lim, T. H., Jeon, Y. M. \& Gong, M. S. Humidity sensors fabricated with photo-curable electrolyte inks using an ink-jet printing technique and their properties. Sens. Actuators B Chem. 130, 594-598 (2008)

160. Thomas, M. S., White, S. P., Dorfman, K. D. \& Frisbie, C. D. Interfacial charge contributions to chemical sensing by electrolyte-gated transistors with floating gates. J. Phys. Chem. Lett. 9, 1335-1339 (2018).

161. Thomas, M. S., Dorfman, K. D. \& Frisbie, C. D. Detection and amplification of capacitance- and charge-based signals using printed electrolyte gated transistors with floating gates. Flexi. Print. Electron. 4 044001 (2019)

162. Lu, B. \& Maharbiz, M. M. Germanium as a scalable sacrificial layer for nanoscale protein patterning. PLOS ONE 13, 1-10 (2018).

163. Holzer, B. et al. Characterization of covalently bound anti-human immunoglobulins on self-assembled monolayer modified gold electrodes. Adv. Biosyst. 1 1700055 (2017).

164. Macchia, E. et al. Single-molecule detection with a millimetre-sized transistor. Nat. Commun. 9, 3223 (2018).

This article is the first demonstration of single molecule detection with a millimetre-sized EGT.

165. Dorfman, K. D., Adrahtas, D. Z., Thomas, M. S. \& Frisbie, C. D. Microfluidic opportunities in printed electrolyte-gated transistor biosensors. Biomicrofluidics 14, 011301 (2020)

166. Curto, V. F., Ferro, M. P., Mariani, F., Scavetta, E. \& Owens, R. M. A planar impedance sensor for 3D spheroids. Lab. Chip 18, 933-943 (2018).

167. Jonkheijm, P., Weinrich, D., Schröder, H., Niemeyer, C. M. \& Waldmann, H. Chemical strategies for generating protein biochips. Angew. Chem. Int. Ed. 47, 9618-9647 (2008)

168. Torsi, L., Magliulo, M., Manoli, K. \& Palazzo, G. Organic field-effect transistor sensors: a tutoria review. Chem. Soc. Rev. 42, 8612-8628 (2013).

169. Macchia, E. et al. Organic bioelectronics probing conformational changes in surface confined proteins. Sci. Rep. 6, 28085 (2016).

170. Shinagawa, T. \& Takanabe, K. Towards versatile and sustainable hydrogen production through electrocatalytic water splitting: electrolyte engineering. ChemSusChem 10, 1318-1336 (2017).

171. Auten, R. L. \& Davis, J. M. Oxygen toxicity and reactive oxygen species: the devil is in the details. Pediatr. Res. 66, 121-127 (2009).

172. Bernards, D. A. \& Malliaras, G. G. Steady-state and transient behavior of organic electrochemical transistors. Adv. Funct. Mater. 17, 3538-3544 (2007)

173. Kireev, D. et al. Graphene transistors for interfacing with cells: towards a deeper understanding of liquid gating and sensitivity. Sci. Rep. 7, 6658 (2017).

174. Friedlein, J. T., McLeod, R. R. \& Rivnay, J. Device physics of organic electrochemical transistors. Org. Electron. 63, 398-414 (2018).
175. Keene, S. T. et al. Enhancement-mode PEDOT:PSS organic electrochemical transistors using molecular de-doping. Adv. Mater. 32, 2000270 (2020)

176. Thiburce, Q., Giovannitti, A., McCulloch, I. \& Campbell, A. J. Nanoscale ion-doped polymer transistors. Nano Lett. 19, 1712-1718 (2019).

177. Braendlein, M., Lonjaret, T., Leleux, P., Badier, J.-M. ¿ Malliaras, G. G. Voltage amplifier based on organic electrochemical transistor. Adv. Sci. 4, 1600247 (2016).

178. Macchia, E. et al. About the amplification factors in organic bioelectronic sensors. Mater. Horiz. 7 , 999-1013 (2020).

This article provides a critical review of organic bioelectronic sensors operating in potentiometric and amperometric mode.

179. Porrazzo, R. et al. Water-gated n-type organic field-effect transistors for complementary integrated circuits operating in an aqueous environment. ACS Omega 2, 1-10 (2017).

180. Khodagholy, D. et al. High transconductance organic electrochemical transistors. Nat. Commun. 4, 2133 (2013).

181. Torricelli, F., Colalongo, L., Raiteri, D., Kovács-Vajna, Z. M. \& Cantatore, E. Ultra-high gain diffusion-driven organic transistor. Nat. Commun. 7 , 10550 (2016).

182. Leydecker, T., Wang, Z. M., Torricelli, F. \& Orgiu, E. Organic-based inverters: basic concepts, materials, novel architectures and applications. Chem. Soc. Rev. 49, 7627-7670 (2020).

183. Paudel, P. R., Kaphle, V., Dahal, D. Radha Krishnan, R. K. \& Lüssem, B. Tuning the transconductance of organic electrochemical transistors. Adv. Funct. Mater. 31, 2004939 (2021).

184. Inal, S. et al. A high transconductance accumulation mode electrochemical transistor. Adv. Mater. 26, 7450-7455 (2014).

185. Di Lauro, M. et al. Liquid-gated organic electronic devices based on high-performance solution-processed molecular semiconductor. Adv. Electron. Mater. 3, 1700159 (2017).

186. Dankerl, M. et al. Graphene solution-gated field-effect transistor array for sensing applications. Adv. Funct. Mater. 20, 3117-3124 (2010)

187. Luan, X., Liu, J. \& Li, H. Electrolyte-gated vertical organic transistor and circuit. J. Phys. Chem C. 122 14615-14620 (2018).

188. Rivnay, J. et al. High-performance transistors for bioelectronics through tuning of channel thickness. Sci. Adv. 1, e 1400251 (2015).

This article is the first demonstration of bioelectronics exploiting the volumetric ionic-electronic charge interaction.

189. Grey, P. et al. Solid state electrochemical $\mathrm{WO}_{3}$ transistors with high current modulation. Adv. Electron. Mater. 2, 1500414 (2016).

190. Bischak, C. G., Flagg, L. O. \& Ginger, D. S. Ion exchange gels allow organic electrochemical transistor operation with hydrophobic polymers in aqueous solution. Adv. Mater. 32, 2002610 (2020).

191. Macchia, E. et al. Ultra-sensitive protein detection with organic electrochemical transistors printed on plastic substrates. Flex. Print. Electron. 3, 1-17 (2018).

192. Kwon, O. S. et al. An ultrasensitive, selective, multiplexed superbioelectronic nose that mimics the human sense of smell. Nano Lett. 15, 6559-6567 (2015).

193. Zhou, L. et al. Novel graphene biosensor based on the functionalization of multifunctional nano-bovine serum albumin for the highly sensitive detection of cancer biomarkers. Nano-Micro Lett. 11, 20 (2019).

194. Murugathas, T. et al. Biosensing with insect odorant receptor nanodiscs and carbon nanotube field-effect transistors. ACS Appl. Mater. Interfaces 11, 9530-9538 (2019)

195. Liang, Y. et al. Wafer-scale uniform carbon nanotube transistors for ultrasensitive and label-free detection of disease biomarkers. ACS Nano 14, 8866-8874 (2020).

196. Macchia, E. et al. Organic field-effect transistor platform for label-free, single-molecule detection of genomic biomarkers. ACS Sens. 5, 1822-1830 (2020).

197. Hess, L. H. et al. Electrical coupling between cells and graphene transistors. Small 11, 1703-1710 (2015).

198. Zhang, Y. et al. Liquid-solid dual-gate organic transistors with tunable threshold voltage for cell sensing. ACS Appl. Mater. Interfaces 9 38687-38694 (2017).
199. Yeung, S. Y., Gu, X., Tsang, C. M., Tsao, S. W. G. \& Hsing, I. Organic electrochemical transistor array for monitoring barrier integrity of epithelial cells invaded by nasopharyngeal carcinoma. Sens. Actuators B Chem. 297, 126761 (2019).

200. Magliulo, M. et al. Electrolyte-gated organic field-effect transistor sensors based on supported biotinylated phospholipid bilayer. Adv. Mater. 25, 2090-2094 (2013).

201. Zhang, Y. et al. Supported lipid bilayer assembly on PEDOT:PSS films and transistors. Adv. Funct. Mater 26, 7304-7313 (2016)

202. Kawan, M. et al. Monitoring supported lipid bilayers with n-type organic electrochemical transistors. Mater. Horiz. 7, 2348-2358 (2020)

203. Magliulo, M. et al. Printable and flexible electronics: from TFTs to bioelectronic devices. J. Mater. Chem. C. 3, 12347-12363 (2015)

204. Nikolka, M. et al. Low-voltage, dual-gate organic transistors with high sensitivity and stability toward electrostatic biosensing. ACS Appl. Mater. Interfaces 12, 40581-40589 (2020)

205. Palazzo, G. et al. Detection beyond Debye's length with an electrolyte-gated organic field-effect transistor Adv. Mater. 27, 911-916 (2015).

206. Aspermair, P. et al. Dual monitoring of surface reactions in real time by combined surface-plasmon resonance and field-effect transistor interrogation. J. Am. Chem. Soc. 142, 11709-11716 (2020).

207. Lieberth, K. et al. Monitoring reversible tight junction modulation with a current-driven organic electrochemical transistor. Adv. Mater. Technol. 6 2000940 (2021)

208. Lieberth, K. et al. Current-driven organic electrochemical transistors for monitoring cell layer integrity with enhanced sensitivity. Adv. Healthcare Mater. https://doi.org/10.1002/adhm.202100845 (2021).

209. Faria, G. C., Duong, D. T. \& Salleo, A. On the transient response of organic electrochemical transistors. Org. Electron. 45, 215-221 (2017).

210. Wang, J. et al. Nanomesh organic electrochemical transistor for comfortable on-skin electrodes with local amplifying function. ACS Appl. Electron. Mater. 2, 3601-3609 (2020)

211. Jo, Y. J. et al. Biocompatible and biodegradable organic transistors using a solid-state electrolyte incorporated with choline-based ionic liquid and polysaccharide. Adv. Funct. Mater. 30, 1909707 (2020)

212. Tyrrell, J. E., Boutelle, M. G. \& Campbell, A. J. Measurement of electrophysiological signals in vitro using high-performance organic electrochemical transistors. Adv. Funct. Mater. 31, 2007086 (2021).

213. Campana, A., Cramer, T., Simon, D. T., Berggren, M. \& Biscarini, F. Electrocardiographic recording with conformable organic electrochemical transistor fabricated on resorbable bioscaffold. Adv. Mater. 26 , 3874-3878 (2014).

214. Yao, C., Li, Q., Guo, J., Yan, F. \& Hsing, I.-M. Rigid and flexible organic electrochemical transistor arrays for monitoring action potentials from electrogenic cells. Adv. Healthcare Mater. 4, 528-533 (2015).

215. Khodagholy, D. et al. NeuroGrid: recording action potentials from the surface of the brain. Nat. Neurosci. 18, 310-315 (2015)

216. Khodagholy, D. et al. In vivo recordings of brain activity using organic transistors. Nat. Commun. $\mathbf{4}$, 1575 (2013).

217. Giaever, I. \& Keese, C. R. Monitoring fibroblast behavior in tissue culture with an applied electric field. Proc. Natl Acad. Sci. USA 81, 3761-3764 (1984).

218. Cereijido, M., Robbins, E. S., Dolan, W. J., Rotunno, C. A $\&$ Sabatini, D. D. Polarized monolayers formed by epithelial cells on a permeable and translucent support. J. Cell Biol. 77, 853-880 (1978).

219. Fromherz, P., Offenhäusser, A., Vetter, T. \& Weis, J. A neuron-silicon junction: a Retzius cell of the leech on an insulated-gate field-effect transistor. Science 252, 1290-1293 (1991)

220. Spira, M. E. \& Hai, A. Multi-electrode array technologies for neuroscience and cardiology. Nat Nanotechnol. 8, 83-94 (2013).

221. Cramer, T. et al. Organic ultra-thin film transistors with a liquid gate for extracellular stimulation an recording of electric activity of stem cell-derived neuronal networks. Phys. Chem. Chem. Phys. 15 3897-3905 (2013).

222. Yao, C. et al. Organic electrochemical transistor array for recording transepithelial ion transport of human airway epithelial cells. Adv. Mater. 25, 6575-6580 (2013). 
223. Gu, X., Yao, C., Liu, Y. \& Hsing, I.-M. 16-Channel organic electrochemical transistor array for in vitro conduction mapping of cardiac action potential. Adv. Healthc. Mater. 5, 2345-2351 (2016).

224. Hess, L. H. et al. Graphene transistor arrays for recording action potentials from electrogenic cells. Adv. Mater. 23, 5045-5049 (2011).

225. Ramuz, M., Hama, A., Rivnay, J., Leleux, P. \& Owens, R. M. Monitoring of cell layer coverage and differentiation with the organic electrochemical transistor. J. Mater. Chem. B 3, 5971-5977 (2015).

226. Ramuz, M. et al. Optimization of a planar all-polymer transistor for characterization of barrier tissue. ChemPhysChem 16, 1210-1216 (2015)

227. Ramuz, M. et al. Combined optical and electronic sensing of epithelial cells using planar organic transistors. Adv Mater 26, 7083-7090 (2014).

228. Curto, V. F. et al. Organic transistor platform with integrated microfluidics for in-line multi-parametric in vitro cell monitoring. Microsyst. Nanoeng. 3, 17028 (2017).

229. Santoro, F. et al. Interfacing electrogenic cells with 3D nanoelectrodes: position, shape, and size matter. ACS Nano 8, 6713-6723 (2014)

230. Kalmykov, A. et al. Organ-on-e-chip: three-dimensional self-rolled biosensor array for electrical interrogations of human electrogenic spheroids. Sci. Adv. 5 , eaax0729 (2019)

231. Wan, A. M.-D. et al. 3D conducting polymer platforms for electrical control of protein conformation and cellular functions. J. Mater. Chem. B 3, 5040-5048 (2015).

232. Inal, S. et al. Conducting polymer scaffolds for hosting and monitoring 3D cell. Culture. Adv. Biosyst. 1 1700052 (2017)

233. Pitsalidis, C. et al. Transistor in a tube: a route to three-dimensional bioelectronics. Sci. Adv. 4 eaat4253 (2018)

This article demonstrates a new route to three-dimensional bioelectronics with EGTs.

234. Moysidou, C.-M. et al. 3D bioelectronic model of the human intestine. Adv. Biol. 5, 2000306 (2021).

235. landolo, D. et al. Biomimetic and electroactive 3D scaffolds for human neural crest-derived stem cell expansion and osteogenic differentiation. MRS Commun. 10, 179-187 (2020).

236. del Agua, I. et al. Conducting polymer scaffolds based on poly(3,4-ethylenedioxythiophene) and xanthan gum for live-cell monitoring. ACS Omega 3 7424-7431 (2018)

237. Gooding, J. J. \& Gaus, K. Single-molecule sensors: challenges and opportunities for quantitative analysis. Angew. Chem. Int. Ed. 55, 11354-11366 (2016).

238. Macchia, E., Manoli, K., Di Franco, C., Scamarcio, G. \& Torsi, L. New trends in single-molecule bioanalytical detection. Anal. Bioanal. Chem. 412, 5005-5014 (2020).

239. Macchia, E. et al. Diffusion of a single marker detected at a large capturing interface. Adv. Sci. (in the press)

240. Rissin, D. M. \& Walt, D. R. Digital concentration readout of single enzyme molecules using femtoliter arrays and poisson statistics. Nano Lett. 6, 520-523 (2006).

241. Park, S. J. et al. Ultrasensitive flexible graphene based field-effect transistor (FET)-type bioelectronic nose. Nano Lett. 12, 5082-5090 (2012)

242. Fu, W. et al. Graphene transistors are insensitive to $\mathrm{pH}$ changes in solution. Nano Lett. 11, 3597-3600 (2011)

243. Macchia, E. et al. Label-free electronic detection of peptides at the physical limit. Adv Healthc. Mater (in the press).

244. Macchia, E. et al. Selective single-molecule analytica detection of C-reactive protein in saliva with an organic transistor. Anal. Bioanal. Chem. 411, 4899-4908 (2019).

245. Buzsáki, G. \& Press., O. U. Rhythms of the Brain (Oxford Univ. Press, 2011).

246. Rogers, J., Malliaras, G. \& Someya, T. Biomedical devices go wild. Sci. Adv. 4, eaav1889 (2018)

247. Strakosas, X., Bongo, M. \& Owens, R. M. The organic electrochemical transistor for biological applications. J. Appl. Polym. Sci. 132, 41735 (2015)

248. Sophocleous, M., Contat-Rodrigo, L., Garcia-Breijo, E. \& Georgiou, J. Organic electrochemical transistors as an emerging platform for bio-sensing applications: a review. IEEE Sens. J. 21, 3977-4006 (2021).

249. San Roman, D., Garg, R. \& Cohen-Karni, T. Bioelectronics with graphene nanostructures. APL. Mater. 8, 100906 (2020).

250. Lee, W. et al. Transparent, conformable, active multielectrode array using organic electrochemical transistors. Proc Natl Acad. Sci. USA 114

10554-10559 (2017)

251. Lee, W. et al. Nonthrombogenic, stretchable, active multielectrode array for electroanatomical mapping. Sci. Adv. 4, eaau2426 (2018).

252. Williamson, A. et al. Localized neuron stimulation with organic electrochemical transistors on delaminating depth probes. Adv. Mater. 27, 4405-4410 (2015).

253. Yang, L. et al. Highly crumpled all-carbon transistor for brain activity recording. Nano Lett. 17, 71-77 (2017).

254. Blaschke, B. M. et al. Mapping brain activity with flexible graphene micro-transistors. 2D Mater. 4 , 025040 (2017).

255. Garcia-Cortadella, R. et al. Switchless multiplexing of graphene active sensor arrays for brain mapping. Nano Lett. 20,3528-3537 (2020).

256. Schaefer, N. et al. Multiplexed neural sensor array of graphene solution-gated field-effect transistors. 2D Mater. 7, 025046 (2020).

257. Garcia-Cortadella, R. et al. Graphene active sensor arrays for long-term and wireless mapping of wide frequency band epicortical brain activity. Nat Commun. 12, 211 (2021).

258. Schuhmann, T. G., Yao, J., Hong, G., Fu, T.-M. \& Lieber, C. M. Syringe-injectable electronics with a plug-and-play input/output interface. Nano Lett. 17, 5836-5842 (2017)

259. Girardi, S., Maschietto, M., Zeitler, R., Mahmud, M $\&$ Vassanelli, S. in Proc. 2011 5th Int. IEEE/EMBS Conf. Neural Eng. 269-272 (IEEE, 2011).

260. Lee, H. et al. Ultrathin organic electrochemical transistor with nonvolatile and thin gel electrolyte for long-term electrophysiological monitoring. Adv. Funct. Mater. 29, 1906982 (2019).

261. Kim, S. J. et al. A new architecture for fibrous organic transistors based on a double-stranded assembly of electrode microfibers for electronic textile applications. Adv. Mater. 31, 1900564 (2019).

262. Leleux, P. et al. Organic electrochemical transistors for clinical applications. Adv. Healthc. Mater. 4, 142-147 (2015).

263. Venkatraman, V. et al. Subthreshold operation of organic electrochemical transistors for biosignal amplification. Adv. Sci. 5, 1800453 (2018).

264. Yu, Y. et al. Small-molecule-based organic field-effect transistor for nonvolatile memory and artificial synapse. Adv. Funct. Mater. 29, 1904602 (2019).

265. Dai, S. et al. Recent advances in transistor-based artificial synapses. Adv. Funct. Mater. 29, 1903700 (2019).

266. Han, H., Yu, H., Wei, H., Gong, J. \& Xu, W. Recent progress in three-terminal artificial synapses: from device to system. Small 15, 1900695 (2019).

267. Strukov, D. B., Snider, G. S., Stewart, D. R. \& Williams, R. S. The missing memristor found. Nature 453, 80-83 (2008).

268. van de Burgt, Y., Melianas, A., Keene, S. T., Malliaras, C. $£$ Salleo, A. Organic electronics for neuromorphic computing. Nat. Electron. 1, 386-397 (2018).

269. Xu, W., Min, S.-Y., Hwang, H. \& Lee, T.-W. Organic core-sheath nanowire artificial synapses with femtojoule energy consumption. Sci. Adv. 2 e 1501326 (2016)

270. van de Burgt, Y. et al. A non-volatile organic electrochemical device as a low-voltage artificial synapse for neuromorphic computing. Nat. Mater. 16, 414-418 (2017).

271. Emelyanov, A. V. et al. First steps towards the realization of a double layer perceptron based on organic memristive devices. AIP Adv. 6, 111301 (2016).

272. Battistoni, S, Erokhin, V \& lannotta, S Emulation with organic memristive devices of impairment of LTP mechanism in neurodegenerative disease pathology. Neural Plast. 2017, 6090312 (2017).

273. Juzekaeva, E. et al. Coupling cortical neurons through electronic memristive synapse. Adv. Mater. Technol. 4 1800350 (2019)

274. Gkoupidenis, P., Schaefer, N., Garlan, B. \& Malliaras, G G. Neuromorphic functions in PEDOT:PSS organic electrochemical transistors. Adv. Mater. 27, 7176-7180 (2015).

275. Gkoupidenis, P., Schaefer, N., Strakosas, X. Fairfield, J. A. \& Malliaras, G. G. Synaptic plasticity functions in an organic electrochemical transistor. Appl. Phys. Lett. 107, 263302 (2015).

276. Gkoupidenis, P., Koutsouras, D. A., Lonjaret, T. Fairfield, J. A. ¿ Malliaras, G. G. Orientation selectivity in a multi-gated organic electrochemical transistor. Sci. Rep. 6, 27007 (2016)
277. Koutsouras, D. A., Malliaras, G. G. \& Gkoupidenis, P. Emulating homeoplasticity phenomena with organic electrochemical devices. MRS Commun. 8, 493-497 (2018).

278. Jörntell, H. et al. Segregation of tactile input features in neurons of the cuneate nucleus. Neuron 83 1444-1452 (2014).

279. Bengtsson, F., Brasselet, R., Johansson, R. S., Arleo, A. $\&$ Jörntell, H. Integration of sensory quanta in cuneate nucleus neurons in vivo. PLOS ONE 8, e56630 (2013).

280. Knibestöl, M Stimulus-response functions of slowly adapting mechanoreceptors in the human glabrous skin area. J. Physiol. 245, 63-80 (1975).

281. Tee, B. C.-K. et al. A skin-inspired organic digital mechanoreceptor. Science 350, 313-316 (2015).

282. Kim, Y. et al. A bioinspired flexible organic artificial afferent nerve. Science 360, 998-1003 (2018). This article is the first demonstration of an artificial afferent nerve with EGTs.

283. Takeuchi, Y. et al. Rewiring of afferent fibers in the somatosensory thalamus of mice caused by peripheral sensory nerve transection. J. Neurosci. 32 6917-6930 (2012).

284. Lee, Y. et al. Stretchable organic optoelectronic sensorimotor synapse. Sci. Adv. 4, eaat7387 (2018).

285. Jung, Y. H., Park, B., Kim, J. U. \& Kim, T. Bioinspired electronics for artificial sensory systems. Adv. Mater. 31, 1803637 (2019)

286. Shim, H. et al. Stretchable elastic synaptic transistors for neurologically integrated soft engineering systems. Sci. Adv. 5, eaax4961 (2019).

287. Park, H.-L. et al. Flexible neuromorphic electronics for computing, soft robotics, and neuroprosthetics. Adv. Mater. 32, 1903558 (2020).

288. Wan, C. et al. Artificial sensory memory. Adv. Mater. 32, 1902434 (2020)

289. Porrazzo, R. et al. Improving mobility and electrochemical stability of a water-gated polymer field-effect transistor. Org. Electron. 15, 2126-2134 (2014).

290. Vagin, M. et al. Negatively-doped conducting polymers for oxygen reduction reaction. Adv. Energy Mater. 11, 2002664 (2021)

291. Picca, R. A. et al. A study on the stability of water-gated organic field-effect-transistors based on a commercial p-type polymer. Front. Chem. 7, 1-10 (2019).

292. Giridharagopal, R. et al. Electrochemical strain microscopy probes morphology-induced variations in ion uptake and performance in organic electrochemical transistors. Nat. Mater. 16, 737-742 (2017).

293. Wang, G. Y., Lian, K. \& Chu, T. Y. Electrolyte-gated field effect transistors in biological sensing: a survey of electrolytes. IEEE J. Electron Devices Soc. https:// doi.org/10.1109/JEDS.2021.3082420 (2021).

294. Park, S. et al. Sub-0.5V highly stable aqueous salt gated metal oxide electronics. Sci. Rep. 5, 1-9 (2015).

295. Bubel, S., Meyer, S. \& Chabinyc, M. L. Stability of ionic liquid-gated metal oxides and transistors. IEEE Trans. Electron. Devices 61, 1561-1566 (2014).

296. Thiemann, S., Sachnov, S., Porscha, S., Wasserscheid, P. $\&$ Zaumseil, J. Ionic liquids for electrolyte-gating of ZnO field-effect transistors. J. Phys. Chem. C. 116, 13536-13544 (2012)

297. van Druenen, M. Degradation of black phosphorus and strategies to enhance its ambient lifetime. Adv. Mater. Interfaces 7, 1-14 (2020).

298. Flagg, L. Q., Giridharagopal, R., Guo, J. \& Ginger, D. S. Anion-dependent doping and charge transport in organic electrochemical transistors. Chem. Mater. 30 5380-5389 (2018).

299. Torricelli, F. et al. in Proc. 2019 Int. Conf. Simulation of Semiconductor Processes and Devices (SISPAD) 1-4 (IEEE, 2019).

300. Robin, M. et al. Overcoming electrochemical instabilities of printed silver electrodes in all-printed ion gel gated carbon nanotube thin-film transistors. ACS Appl. Mater. Interfaces 11, 41531-41543 (2019).

301. Prescimone, F. et al. 3D versus 2D electrolytesemiconductor interfaces in rylenediimide-based electron-transporting water-gated organic field-effect transistors. Adv. Electron. Mater. 6, 2-11 (2020)

302. Torricelli, F. Enhanced multifunctional bioelectronics with integrated organic electrochemical transistor architectures. SPIE 11663, 116630P (2021).

303. Paterson, A. F. et al. Water stable molecular n-doping produces organic electrochemical transistors with high transconductance and record stability. Nat. Commun. $11,3004(2020)$ 
304. Torsi, L. et al. A sensitivity-enhanced field-effect chiral sensor. Nat. Mater 7 412-417 (2008).

305. Macchia, E., Giordano, F., Magliulo, M., Palazzo, G. \& Torsi, L. An analytical model for bio-electronic organic field-effect transistor sensors. Appl. Phys. Lett. 103 103301 (2013)

306. Magliulo, M. et al. PE-CVD of hydrophilic- $\mathrm{COOH}$ functionalized coatings on electrolyte gated field-effect transistor electronic layers. Plasma Process. Polym. 10, 102-109 (2013).

307. Giovannitti, A. et al. Controlling the mode of operation of organic transistors through side-chain engineering. Proc. Natl Acad. Sci. USA 113, 12017-12022 (2016).

308. Suspène, C. et al. Copolythiophene-based water-gated organic field-effect transistors for biosensing. J. Mater Chem. B 1, 2090-2097 (2013).

309. Angione, M. D. et al. Interfacial electronic effects in functional biolayers integrated into organic fieldeffect transistors. Proc. Natl Acad. Sci. USA 109, 6429-6434 (2012).

310. Pappa, A. M. et al. Optical and electronic ion channel monitoring from native human membranes. ACS Nano 14, 12538-12545 (2020).

311. Liu, H. Y. et al. Self-assembly of mammalian-cell membranes on bioelectronic devices with functional transmembrane proteins. Langmuir 36, 7325-733 (2020).

312. Cui, B. et al. Thermal conductivity comparison of indium gallium zinc oxide thin films: dependence on temperature, crystallinity, and porosity. J. Phys. Chem C. 120,7467-7475 (2016)

313. Li, H., Guo, Y. \& Robertson, J. Hydrogen and the lightinduced bias instability mechanism in amorphous oxide semiconductors. Sci. Rep. 7, 16858 (2017)

314. Medvedeva, J. E., Buchholz, D. B. \& Chang, R. P. H. Recent advances in understanding the structure and properties of amorphous oxide semiconductors. Adv. Electron. Mater. 3, 1700082 (2017)

315. Armano, A. \& Agnello, S. Two-dimensional carbon a review of synthesis methods, and electronic, optical, and vibrational properties of single-layer. Graphene. $C$. 5, 67 (2019).

316. He, Z. \& Que, W. Molybdenum disulfide nanomaterials: structures, properties, synthesis and recent progress on hydrogen evolution reaction. Appl. Mater. Today 3, 23-56 (2016).

317. Casalini, S., Leonardi, F., Cramer, T. \& Biscarini, F. Organic field-effect transistor for label-free dopamine sensing. Org. Electron. Phys. Mater. Appl. 14 156-163 (2013)

318. Blasi, D. et al. Enhancing the sensitivity of biotinylated surfaces by tailoring the design of the mixed selfassembled monolayer synthesis. ACS Omega 5 16762-16771 (2020).

319. Casalini, S. et al. Multiscale sensing of antibody antigen interactions by organic transistors and single-molecule force spectroscopy. ACS Nano 9 5051-5062 (2015) This article provides fundamental insights into antigen-antibody interactions.
320. Berto, M. et al. Label free detection of plant viruses with organic transistor biosensors. Sens. Actuators B Chem. 281, 150-156 (2019).

321. Selvaraj, M. et al. Label free detection of miRNA-21 with electrolyte gated organic field effect transistors (EGOFETs). Biosens. Bioelectron. 182, 113144 (2021).

322. Sensi, M. et al. Anti-drug antibody detection with label-free electrolyte-gated organic field-effect transistors. Chem. Commun. 57, 367-370 (2021).

323. Helmholtz, H. Ueber einige gesetze der vertheilung elektrischer ströme in körperlichen leitern mit anwendung auf die thierisch-elektrischen versuche [German]. Ann. Phys. 165, 211-233 (1853).

324. Stern, O. Zur theorie der elektrolytischen doppelschicht [German]. Z. für Elektrochemie und Angew. Phys. Chem 30, 508-516 (1924)

325. Berggren, M. \& Malliaras, G. G. How conducting polymer electrodes operate. Science 364, 233-234 (2019).

326. Bisquert, J. et al. A review of recent results on electrochemical determination of the density of electronic states of nanostructured metal-oxide semiconductors and organic hole conductors. Inorganica Chim. Acta 361, 684-698 (2008).

327. Lee, J. et al. Ion gel-gated polymer thin-film transistors: operating mechanism and characterization of gate dielectric capacitance, switching speed, and stability. J. Phys. Chem. C. 113, 8972-8981 (2009).

328. Yãñez-Sedeño, P., Pingarrón, J. M., Riu, J. \& Rius, F. X Electrochemical sensing based on carbon nanotubes. Trends Analyt. Chem. 29, 939-953 (2010).

329. Yuan, H. et al. Electrostatic and electrochemical nature of liquid-gated electric-double-layer transistors based on oxide semiconductors. J. Am. Chem. Soc. 132 18402-18407 (2010).

330. Laiho, A., Herlogsson, L., Forchheimer, R., Crispin, X. $\&$ Berggren, M. Controlling the dimensionality of charge transport in organic thin-film transistors. Proc. Natl Acad. Sci. USA 108, 15069-15073 (2011).

331. Vagin, M., Che, C., Gueskine, V., Berggren, M. \& Crispin, X. Ion-selective electrocatalysis on conducting polymer electrodes: improving the performance of redox flow batteries. Adv. Funct. Mater. 30, 2007009 (2020).

332. Mackin, C et al. A current-voltage model for graphene electrolyte-gated field-effect transistors. IEEE Trans. Electron. Devices 61, 3971-3977 (2014).

333. Sahalianov, I., Singh, S. K., Tybrandt, K., Berggren, M $\&$ Zozoulenko, I. The intrinsic volumetric capacitance of conducting polymers: pseudo-capacitors or doublelayer supercapacitors? RSC Adv, 9, 42498-42508 (2019).

334. Singh, M. et al. Effect of the gate metal work function on water-gated $\mathrm{ZnO}$ thin-film transistor performance. J. Phys. D Appl. Phys. 49, 275101 (2016).

335. Lai, S et al. Ultralow voltage, OTFT-based sensor for label-free DNA detection. Adv. Mater. 25, 103-107 (2013).
336. Napoli, C et al. Electronic detection of DNA hybridization by coupling organic field-effect transistor-based sensors and hairpin-shaped probes. Sensors 18, 990 (2018)

337. Spanu, A., Tedesco, M. T., Martines, L., Martinoia, S. $\&$ Bonfiglio, A. An organic neurophysiological tool for neuronal metabolic activity monitoring. APL. Bioeng. 2, 046105 (2018)

338. Spanu, A. et al. A reference-less $\mathrm{pH}$ sensor based on an organic field effect transistor with tunable sensitivity. Org. Electron. 48, 188-193 (2017).

339. Spanu, A. et al. A high-sensitivity tactile sensor based on piezoelectric polymer PVDF coupled to an ultra-low voltage organic transistor. Org. Electron. Phys. Mater Appl. 36, 57-60 (2016).

340. Viola, F. A., Spanu, A., Ricci, P. C., Bonfiglio, A. \& Cosseddu, P. Ultrathin, flexible and multimodal tactile sensors based on organic field-effect transistors. Sci. Rep. 8, 8073 (2018).

\section{Acknowledgements}

F.T and L.T. acknowledge financial support from the European Union, Italian Government and Lombardia Region for the project BIOSCREEN (POR FESR 2014-2020, ID number 1831459, CUP E81B20000320007). F.T., E.M. and L.T. acknowledge financial support from the European Commission for the project SiMBiT (Horizon 2020 ICT, contract number 824946 ). D.Z.A. was supported by a Biotechnology Training (Grant No. NIH T32GM008347). G.G.M. acknowledges support from H2020-EU-FET Open MITICS (964677).

\section{Author contributions}

Introduction (F.T., C.D.F. and D.Z.A.); Box 1 (F.B. and C.A.B.); Experimentation (T.-Q.N., I.M., M.M., C.D.F., D.Z.A., L.T., E.M., F.B. and C.A.B.); Box 2 (M.B.), Box 3 (A.B. and A. Spanu); Results (F.T.); Applications (R.M.O., L.T., G.G.M., A. Salleo and Z.B); Reproducibility and data deposition (F.T. and G.G.M.); Limitations and optimizations (F.T., F.B. and C.A.B.); Outlook (L.T.); Overview of the Primer (F.T. and L.T.). All authors commented on the paper.

\section{Competing interests}

The authors declare no competing interests.

\section{Peer review information}

Nature Reviews Methods Primers thanks S. Dasgupta, H. Kawarada, Q. Wan and the other, anonymous, reviewer(s) for their contribution to the peer review of this work.

\section{Publisher's note}

Springer Nature remains neutral with regard to jurisdictional claims in published maps and institutional affiliations.

\section{Supplementary information}

The online version contains supplementary material available at https://doi.org/10.1038/s43586-021-00065-8.

(C) Springer Nature Limited 2021 